\title{
Breakup of three particles within the adiabatic expansion method
}

\author{
E. Garrido \\ Instituto de Estructura de la Materia, CSIC, Serrano 123, E-28006 Madrid, Spain
}

A. Kievsky and M. Viviani

Istituto Nazionale di Fisica Nucleare, Largo Pontecorvo 3, 56100 Pisa, Italy

(Received 6 May 2014; published 18 July 2014)

\begin{abstract}
General expressions for the breakup cross sections in the laboratory frame for $1+2$ reactions are given in terms of the hyperspherical adiabatic basis. The three-body wave function is expanded in this basis and the corresponding hyperradial functions are obtained by solving a set of second order differential equations. The $\mathcal{S}$ matrix is computed by using two recently derived integral relations. Even though the method is shown to be well suited to describe $1+2$ processes, there are particular configurations in the breakup channel (for example, those in which two particles move away close to each other in a relative zero-energy state) that need a huge number of basis states. This pathology manifests itself in the extremely slow convergence of the breakup amplitude in terms of the hyperspherical harmonic basis used to construct the adiabatic channels. To overcome this difficulty the breakup amplitude is extracted from an integral relation as well. For the sake of illustration, we consider neutron-deuteron scattering. The results are compared to the available benchmark calculations.
\end{abstract}

DOI: 10.1103/PhysRevC.90.014607

PACS number(s): 25.10.+s, 03.65.Nk, 31.15.xj

\section{INTRODUCTION}

The use of the hyperspherical adiabatic (HA) expansion method [1] to describe a $1+2$ collision between a particle and a bound state of two particles, called a dimer in atomic physics or a deuteron in nuclear physics, was considered to be rather inefficient due to the slow pattern of convergence of the elastic channel [2]. This fact seemed to limit the applicability of the method only to the description of bound states. The problem is due to the fact that the hyperradial coordinate used in the adiabatic expansion is not suitable for describing the asymptotic behavior of the elastic outgoing wave. In this case the use of the relative coordinate between the two particles in the outgoing dimer, and the one between the center of mass of the dimer and the third particle is much more convenient. In particular, due to the finite size of the dimer, the latter relative coordinate and the hyperradius coincide only at infinity. For this reason, the correct description of the asymptotic $1+2$ wave function and, therefore, the determination of the $\mathcal{S}$-matrix require knowledge of the wave function at very large distances, which in turn requires a huge number of terms in the adiabatic expansion [3].

A new method, based on two integral relations derived from the Kohn variational principle (KVP), was introduced in [3] in order to permit the determination of the elastic $\mathcal{S}$ matrix from the internal part of the wave function. Therefore, when used together with the adiabatic expansion method, the number of adiabatic terms needed in the calculation is much lower. In fact, the pattern of convergence is similar to that observed for bound states [3]. The details of the procedure for elastic, inelastic, and recombination processes are given in Refs. [4] and [5] for energies below the dimer breakup threshold. The extension to treat the elastic channel at energies above the breakup threshold was discussed in Ref. [6].

The knowledge of the elastic, inelastic, and recombination $\mathcal{S}$-matrix elements can be used to compute different observables characterizing the reaction. Furthermore, using the unitary condition

$$
\sum_{m}^{M}\left|S_{i m}\right|^{2}+\sum_{n}^{\infty}\left|\mathcal{S}_{i n}\right|^{2}=1,
$$

where $m$ indicates the finite set $M$ of elastic, inelastic, and recombination channels and $n$ labels the infinite set of breakup channels, it is possible to obtain the total breakup cross section

$$
\sigma_{b}=\frac{\pi}{k^{2}}\left(1-\sum_{m}^{M}\left|S_{i m}\right|^{2}\right),
$$

where, for three particles with equal mass, we have that $k^{2}=(4 / 3) E_{i}\left(\hbar^{2} / m\right)$ and $E_{i}$ is the incident energy in the center-of-mass frame. Examples of this procedure can be found in Refs. [6] and [7], where the inelasticities in $n-d$ scattering have been computed, as well as the recombination and dissociation rates in the direct and inverse atomic processes ${ }^{4} \mathrm{He}+{ }^{4} \mathrm{He}+{ }^{4} \mathrm{He} \rightarrow{ }^{4} \mathrm{He}_{2}+{ }^{4} \mathrm{He}$. Therefore, an accurate calculation of the elastic and, if allowed, inelastic and recombination $\mathcal{S}$-matrix elements leads to an accurate determination of the corresponding total breakup cross section through the unitary condition.

On the other hand, when knowledge of the breakup amplitude is required, the $S_{i n}$-matrix elements have to be computed explicitly. Using the HA method, the index $n$ is related to the number of adiabatic channels taken into account in the description of the three-body scattering wave function. In the present work each adiabatic channel is expanded in the hyperspherical harmonic $(\mathrm{HH})$ basis. Therefore a study of the convergence properties of the breakup amplitude in terms of the adiabatic channels and the $\mathrm{HH}$ basis is in order. These two convergencies have to be achieved separately. As we will see, the number of adiabatic channels needed to describe the elastic channel with a high accuracy is sufficient for an accurate 
determination of the breakup amplitude. However, there are particular kinematic conditions of the outgoing particles in which the convergence of the breakup amplitude is terms of the $\mathrm{HH}$ basis becomes very delicate. This is the case when two of the particles move away close to each other with almost-zero relative energy. In order to treat this specific configuration we make use of an integral relation for the breakup amplitude as discussed, for example, in Ref. [8].

The method discussed in the present work is general and can be applied to different kinds of three-body reactions. In this work we consider the $N-d$ case, which is frequently described using the Faddeev equations, as shown, for instance, in Refs. [9] and [10] or in the recent review in Ref. [11], or using the HH formalism in conjunction with the KVP [12]. Calculation using the Faddeev equations in momentum space including the Coulomb force between the two protons can be found in Ref. [13]. This and the HH method have been compared in the elastic channel up to $65 \mathrm{MeV}$ [14]. Attempts to explicitly determine the breakup amplitude using the $\mathrm{HH}$ formalism can be found in Refs. [15] and [16], whereas the formalism using the KVP is discussed in Ref. [17]. Those calculations have shown the intrinsic difficulties of a variational description of the breakup amplitude. As mentioned above, the main problem appears in the description of particular kinematics such as, for example, the case in which two particles, for instance, two neutrons, travel close to each other in a relative zero-energy state. This configuration represents a kind of clusterization inside the breakup amplitude and requires a huge number of basis states to be described properly.

Using the experience gained in Refs. [4-6], in which the HA method was used to describe elastic, inelastic, and recombination processes by means of two integral relations derived from the KVP, in the present work we extend the HA method to describe the breakup amplitude explicitly. Expressions for the differential cross sections in the laboratory frame for $1+2$ reactions at incident energies above the dimer breakup threshold are derived. The neutron-deuteron $(n-d)$ reaction is studied with a semirealistic $s$-wave force for illustration. This choice is motivated by the existence of benchmark calculations [18] which allow a test of the method. This study is a first step in the application of the method to describe $N$ - $d$ scattering using realistic two- and three-body forces and including the Coulomb interaction.

In the first part of the paper we provide the details of the formalism used to compute the differential cross sections. This part is divided into several subsections where we give the expression of the cross section in the laboratory frame, the expansion of the transition amplitude in terms of HA functions, and, finally, an integral relation to compute the transition amplitude which removes the convergence problem inherent to the HA expansion. In Sec. III the computation of the integral relation is discussed, in particular, the treatment of the long tail of the kernel. The results obtained for the case of $n$ - $d$ breakup are described in Sec. IV and the last section is devoted to some conclusive remarks. For the sake of completeness, the paper includes six Appendices in which some derivations not essential for the understanding of the paper are given. In particular, several technical aspects of the scattering theory are discussed in terms of the HA expansion method.

\section{FORMALISM}

In this section we summarize the formalism employed to compute the differential cross sections for $1+2$ reactions at energies above the breakup threshold. To this end, we have divided the section into four parts, which correspond to

(i) description of the notation used;

(ii) derivation of the general expression of the cross section in the laboratory frame in terms of the transition amplitude;

(iii) expansion of the transition amplitude in terms of the HA functions; and

(iv) derivation of the integral relation in terms of the three-nucleon scattering wave function in which the outgoing six-dimensional wave is not expanded in the HA basis.

This relation can correct some inaccuracies in the computed transition amplitude (see, for example, Ref. [8]). It also manifests the variational character of the method. The details of how to compute this matrix element and, in particular, how to compute the long tail of the integral contained in this matrix element are discussed in the next section. Some theoretical derivations, not crucial for an understanding of the formalism, but in order to have a compact presentation of the method, have been collected in the Appendices.

\section{A. Notation and coordinates}

Let us denote by $\boldsymbol{r}_{i}(i=1,2,3)$ the coordinates of the three particles involved in the $1+2$ reaction under investigation and by $\boldsymbol{p}_{i}(i=1,2,3)$ their corresponding momenta. From these coordinates we construct the usual Jacobi coordinates, which are given by

$$
\begin{aligned}
& \boldsymbol{x}_{i}=\sqrt{\frac{\mu_{x_{i}}}{m}}\left(\boldsymbol{r}_{j}-\boldsymbol{r}_{k}\right)=\sqrt{\frac{\mu_{x_{i}}}{m}} \boldsymbol{r}_{x_{i}}, \\
& \boldsymbol{y}_{i}=\sqrt{\frac{\mu_{y_{i}}}{m}}\left(\boldsymbol{r}_{i}-\frac{m_{j} \boldsymbol{r}_{j}+m_{k} \boldsymbol{r}_{k}}{m_{j}+m_{k}}\right)=\sqrt{\frac{\mu_{y_{i}}}{m}} \boldsymbol{r}_{y_{i}},
\end{aligned}
$$

where $\mu_{x_{i}}$ is the reduced mass of the $j k$ two-body system, $\mu_{y_{i}}$ is the reduced mass of particle $i$ and the two-body system $j k, m$ is an arbitrary normalization mass, and $m_{i}(i=1,2,3)$ are the masses of the three particles. Cyclic permutations of $\{i, j, k\}$ give the three possible sets of Jacobi coordinates.

The corresponding Jacobi coordinates in momentum space take the form

$$
\begin{aligned}
\boldsymbol{k}_{x_{i}} & =\sqrt{\frac{m}{\mu_{x_{i}}}}\left(\frac{m_{k}}{m_{j}+m_{k}} \boldsymbol{p}_{j}-\frac{m_{j}}{m_{j}+m_{k}} \boldsymbol{p}_{k}\right) \\
& =\sqrt{\frac{m}{\mu_{x_{i}}}} \boldsymbol{p}_{x_{i}}, \\
\boldsymbol{k}_{y_{i}} & =\sqrt{\frac{m}{\mu_{y_{i}}}}\left(\frac{\left(m_{j}+m_{k}\right) \boldsymbol{p}_{i}}{m_{i}+m_{j}+m_{k}}-\frac{m_{i}\left(\boldsymbol{p}_{j}+\boldsymbol{p}_{k}\right)}{m_{i}+m_{j}+m_{k}}\right) \\
& =\sqrt{\frac{m}{\mu_{y_{i}}}} \boldsymbol{p}_{y_{i}} .
\end{aligned}
$$


From the Jacobi coordinates we construct the hyperspherical coordinates. They are given by one radial coordinate, the hyperradius $\rho$, defined as $\rho=\sqrt{x^{2}+y^{2}}$ (the definition is independent of the Jacobi set used), and five hyperangles, which are given by $\alpha_{i}=\arctan x_{i} / y_{i}$ and the polar and azimuthal angles describing the direction of $\boldsymbol{x}_{i}$ and $\boldsymbol{y}_{i}$, i.e., $\Omega_{x_{i}} \equiv\left\{\theta_{x_{i}}, \varphi_{x_{i}}\right\}$ and $\Omega_{y_{i}} \equiv\left\{\theta_{y_{i}}, \varphi_{y_{i}}\right\}$. The hyperangles depend on the Jacobi set chosen to describe the three-body system, and we denote them in a compact form as $\Omega_{i} \equiv\left\{\alpha_{i}, \Omega_{x_{i}}, \Omega_{y_{i}}\right\}$.

The corresponding hyperspherical coordinates in momentum space are given by the three-body momentum $\kappa=$ $\sqrt{k_{x}^{2}+k_{y}^{2}}$ and the five hyperangles $\Omega_{\kappa_{i}} \equiv\left\{\alpha_{\kappa_{i}}, \Omega_{k_{x_{i}}}, \Omega_{k_{y_{i}}}\right\}$, where $\alpha_{\kappa_{i}}=\arctan k_{x_{i}} / k_{y_{i}}$. The three-body momentum $\kappa$ is related to the total three-body energy $E$ of the process by the expression $\kappa=\sqrt{2 m E} / \hbar$.

Note that the volume element is given in terms of the relative coordinates $\boldsymbol{r}_{x_{i}}$ and $\boldsymbol{r}_{y_{i}}$ defined in Eqs. (3) and (4). Therefore,

$$
\begin{aligned}
d V_{i}=d \boldsymbol{r}_{x_{i}} d \boldsymbol{r}_{y_{i}} & =\left(\frac{m}{\mu_{x_{i}}}\right)^{3 / 2}\left(\frac{m}{\mu_{y_{i}}}\right)^{3 / 2} d \boldsymbol{x}_{i} d \boldsymbol{y}_{i} \\
& =\left(\frac{m}{\mu_{x_{i}}}\right)^{3 / 2}\left(\frac{m}{\mu_{y_{i}}}\right)^{3 / 2} \rho^{5} d \rho d \Omega_{i},
\end{aligned}
$$

where $d \Omega_{i}=\sin ^{2} \alpha_{i} \cos ^{2} \alpha_{i} d \alpha_{i} d \Omega_{x_{i}} d \Omega_{y_{i}}$, which means that the hypersurface element of the hypersphere with hyperradius $\rho$ is given by

$$
d \Sigma_{i}=\left(\frac{m}{\mu_{x_{i}}}\right)^{3 / 2}\left(\frac{m}{\mu_{y_{i}}}\right)^{3 / 2} \rho^{5} d \Omega_{i} .
$$

It is important to note that asymptotically the hyperangles in coordinate $\left(\Omega_{i}\right)$ and momentum $\left(\Omega_{\kappa_{i}}\right)$ space coincide. This is related to the fact that the $\mathrm{HHs}$ transform into themselves after a Fourier transformation. A more intuitive way of checking this fact is that asymptotically, at a given time $t$, the coordinate of particle $i$ is just given by $\boldsymbol{r}_{i} \rightarrow t \boldsymbol{p}_{i} / m_{i}$. When replacing these expressions for $\boldsymbol{r}_{i}, \boldsymbol{r}_{j}$, and $\boldsymbol{r}_{k}$ in Eqs. (3) and (4), and taking into account definitions (5) and (6), we immediately get that $\boldsymbol{x}_{i} \rightarrow$ $t \boldsymbol{k}_{x_{i}} / m$ and $\boldsymbol{y}_{i} \rightarrow t \boldsymbol{k}_{y_{i}} / m$. Therefore, asymptotically, the polar and azimuthal angles describing the directions of $\boldsymbol{x}_{i}$ and $\boldsymbol{y}_{i}$ are the same as those describing the directions of $\boldsymbol{k}_{x_{i}}$ and $\boldsymbol{k}_{y_{i}}$, and also, $x_{i} / y_{i}=k_{x_{i}} / k_{y_{i}}$, which implies that, asymptotically, $\alpha_{i}=\alpha_{\kappa_{i}}$. Therefore, asymptotically, $d \Omega_{i}=d \Omega_{\kappa_{i}}$.

When describing the incoming $1+2$ channel it is convenient to choose the Jacobi set such that the relative coordinate $\boldsymbol{r}_{x}$ in Eq. (3) is the relative coordinate between the two particles in the dimer. In this case the Jacobi momentum $\boldsymbol{k}_{y}$ in Eq. (6) is given by $\boldsymbol{k}_{y}=\sqrt{m / \mu_{y}} \boldsymbol{p}_{y}$, where $\boldsymbol{p}_{y}$ is just the incident relative projectile-dimer momentum in the center-of-mass frame. We denote these two vectors, $\boldsymbol{k}_{y}$ and $\boldsymbol{p}_{y}$, as $\boldsymbol{k}_{y}^{(\mathrm{in})}$ and $\boldsymbol{p}_{y}^{(\mathrm{in})}$, such that they can be distinguished from the corresponding momenta in the final state. The momentum $\boldsymbol{p}_{y}^{(\mathrm{in})}$ is related to the incident energy $E_{\text {in }}$ (in the center-of-mass frame) by the expression $p_{y}^{(\text {in })}=\sqrt{2 \mu_{y} E_{\text {in }}} / \hbar$, and the total energy $E$ is then given by $E=E_{\text {in }}+E_{d}$, where $E_{d}$ is the binding energy of the dimer $\left(E_{d}<0\right)$. In the following, unless explicitly mentioned, we use this Jacobi set (the dimer wave function depends only on the $\boldsymbol{x}$ coordinate) and we omit the index $i$ when referring to the $(\boldsymbol{x}, \boldsymbol{y})$ or $\left(\boldsymbol{k}_{x}, \boldsymbol{k}_{y}\right)$ coordinates defined in Eqs. (3) to (6).

\section{B. Breakup cross section in the laboratory frame}

The differential cross section $d \sigma$ after a $1+2$ breakup reaction is given by the outgoing flux of the particles through an element of the hypersurface, Eq. (8), normalized with the incident flux. The expression for the outgoing flux is derived in Appendix A, and it is given by Eq. (A7):

$$
\text { outgoing flux }=\hbar \frac{\kappa}{m}\left|A_{\sigma_{d} \sigma_{p}}^{\sigma_{i} \sigma_{j} \sigma_{k}}\right|^{2}\left(\frac{m}{\mu_{x}}\right)^{3 / 2}\left(\frac{m}{\mu_{y}}\right)^{3 / 2} d \Omega_{\kappa},
$$

where $A_{\sigma_{d} \sigma_{p}}^{\sigma_{i} \sigma_{j} \sigma_{k}}$ is the breakup transition amplitude, in which we have made explicit the spin projections $\sigma_{i}, \sigma_{j}, \sigma_{k}, \sigma_{d}$, and $\sigma_{p}$, which correspond to the three particles found after the breakup (with spins $s_{i}, s_{j}$, and $s_{k}$ ) and to the dimer (with spin $s_{d}$ ) and the projectile (with spin $s_{p}$ ).

The incoming flux is the one corresponding to a particledimer two-body process, and it is given by

$$
\text { incoming flux }=\hbar \frac{p_{y}^{(\mathrm{in})}}{\mu_{y}}=\hbar \sqrt{\frac{m}{\mu_{y}}} \frac{k_{y}^{(\mathrm{in})}}{m},
$$

where the connection between incident momentum $p_{y}^{(\mathrm{in})}$ and $k_{y}^{(\mathrm{in})}$ is given in Eq. (6). The ratio between Eqs. (9) and (10) gives, then, the differential cross section in the three-body center-of-mass frame, and it takes the form

$$
\begin{aligned}
\frac{d^{5} \sigma}{d \Omega_{\kappa}}= & \frac{\kappa}{k_{y}^{(\mathrm{in})}} \sqrt{\frac{\mu_{y}}{m}}\left(\frac{m}{\mu_{x}}\right)^{3 / 2}\left(\frac{m}{\mu_{y}}\right)^{3 / 2} \\
& \times \frac{1}{\left(2 s_{d}+1\right)\left(2 s_{p}+1\right)} \sum_{\sigma_{i} \sigma_{j} \sigma_{k} \sigma_{d} \sigma_{p}}\left|A_{\sigma_{d} \sigma_{p}}^{\sigma_{i} \sigma_{j} \sigma_{k}}\right|^{2},
\end{aligned}
$$

where we have already averaged over the initial states and summed over all the possible final states. This procedure gives the $1 /\left(2 s_{d}+1\right)\left(2 s_{p}+1\right)$ factor and the summation over all the spin projections.

In Appendix B we have derived the phase space in terms of the center-of-mass coordinates, Eq. (B5), and in terms of the laboratory coordinates, Eq. (B24). For simplicity, the derivation of Eq. (B24) has been made assuming three particles with equal mass $m$ [which is also taken to be the normalization mass in Eqs. (3) to (6)]. Thus the expression below is valid only for this particular case, although the generalization to three particles with different masses is straightforward. Making equal Eqs. (B5) and (B24) we then obtain

$$
\frac{d \Omega_{\kappa}}{d S d \hat{\boldsymbol{p}}_{i} d \hat{\boldsymbol{p}}_{j}}=\frac{m}{\hbar^{2}}\left(\frac{m}{\mu_{x}}\right)^{3 / 2}\left(\frac{m}{\mu_{y}}\right)^{3 / 2} \frac{K_{S}}{\kappa^{4}},
$$

where $i$ and $j$ refer to two of the outgoing particles, $d \hat{\boldsymbol{p}}_{i}=$ $\sin \theta_{p_{i}} d \theta_{p_{i}} d \varphi_{p_{i}},\left\{\theta_{p_{i}}, \varphi_{p_{i}}\right\}$ are the polar and azimuthal angles giving the direction of momentum $\boldsymbol{p}_{i}$ (and similarly for particle $j$ ), the arclength $S$ is defined by Eq. (B21), and, finally, $K_{S}$ is 
given by Eq. (B25). Since

$$
\frac{d^{5} \sigma}{d S d \hat{\boldsymbol{p}}_{i} d \hat{\boldsymbol{p}}_{j}}=\frac{d \Omega_{\kappa}}{d S d \hat{\boldsymbol{p}}_{i} d \hat{\boldsymbol{p}}_{j}} \frac{d^{5} \sigma}{d \Omega_{\kappa}},
$$

we get the following final expression for the cross section in the laboratory frame:

$$
\frac{d^{5} \sigma}{d S d \hat{\boldsymbol{p}}_{i} d \hat{\boldsymbol{p}}_{j}}=\left(\frac{m}{\mu_{x}}\right)^{3 / 2}\left(\frac{m}{\mu_{y}}\right)^{3 / 2} \frac{m}{\hbar^{2}} \frac{K_{S}}{\kappa^{4}} \frac{d^{5} \sigma}{d \Omega_{\kappa}},
$$

where $d^{5} \sigma / d \Omega_{\kappa}$ is given in Eq. (11).

In this work we focus on $n-d$ breakup reactions. For this case we have three spin-1/2 particles with mass $m$ equal to the nucleon mass, such that the spins of the dimer and the projectile are, respectively, $s_{d}=1$ and $s_{p}=1 / 2$. Also, $k_{y}^{\text {(in) }}$ is given by $\sqrt{3 / 2} p_{y}^{(\mathrm{in})}$, where $p_{y}^{(\mathrm{in})}$ is the relative momentum between projectile and dimer. Then using Eqs. (11) and (14) we obtain for the $n-d$ case,

$$
\frac{d^{5} \sigma}{d S d \hat{\boldsymbol{p}}_{i} d \hat{\boldsymbol{p}}_{j}}=\frac{3 m}{\hbar^{2}} \frac{K_{S}}{\kappa^{3} p_{y}^{(\mathrm{in})}} \sum_{\sigma_{i} \sigma_{j} \sigma_{j}} \sum_{\sigma_{d} \sigma_{p}}\left|A_{\sigma_{d} \sigma_{p}}^{\sigma_{i} \sigma_{j} \sigma_{k}}\right|^{2} .
$$

In our calculations particles $i$ and $j$ in the equation above will be taken to be the two outgoing neutrons. The input will be the neutron incident energy in the laboratory frame $\left[E_{\mathrm{in}}^{(\mathrm{lab})}\right]$, the polar angles $\theta_{p_{i}}$ and $\theta_{p_{j}}$ for the two outgoing neutrons, and $\Delta \varphi=\varphi_{p_{i}}-\varphi_{p_{j}}$. These angles, as shown in Eqs. (B11)(B13), determine the values of $\mu_{i}, \mu_{j}$, and $\mu$ entering into $K_{S}$ [Eq. (B25)].

The input incident energy $E_{\text {in }}^{(\text {lab })}$ immediately provides the momentum of the projectile in the laboratory frame,

$$
p_{y}^{(\mathrm{lab})}=\sqrt{2 m E_{\mathrm{in}}^{(\mathrm{lab})}} / \hbar,
$$

which also enters into $K_{S}$. The laboratory energy $E_{\text {in }}^{(\text {lab) }}$ can be easily related to the incident energy in the center-of-mass frame $\left(E_{\text {in }}\right)$, which, for the case of the $n-d$ reaction, becomes $E_{\mathrm{in}}=2 E_{\mathrm{in}}^{\text {(lab) }} / 3$. From it we can get the center-of-mass relative momentum $p_{y}^{\text {(in) }}$ entering into Eq. (15), which is given by $p_{y}^{(\text {in })}=\sqrt{2 \mu_{y} E_{\text {in }}} / \hbar$, where $\mu_{y}=2 m / 3$ is the projectile-dimer reduced mass.

The cross section given in Eq. (15) is a function of the arclength $S$. For each value of $S$, given the input $E_{\text {in }}^{(\text {lab })}, \theta_{p_{i}}$, $\theta_{p_{j}}$, and $\Delta \varphi$, the values of $p_{i}$ and $p_{j}$ are uniquely determined, as shown in Appendix C. These two momenta, together with the already known values of $p_{y}^{(\text {lab })}, \mu_{i}, \mu_{j}$, and $\mu$, permit us now to compute $K_{S}$ according to Eq. (B25) and then obtain the differential cross section. The only piece remaining is the determination of the breakup transition amplitude $A_{\sigma_{d} \sigma_{p} \sigma_{p}}^{\sigma_{\sigma} \sigma_{k}}$, which is discussed in the following sections.

\section{The breakup transition amplitude in the HA expansion method}

When using hyperspherical coordinates the three-body Hamiltonian operator $\hat{\mathcal{H}}$ takes the form [1]

$$
\hat{\mathcal{H}}=-\frac{\hbar^{2}}{2 m} \hat{T}_{\rho}+\hat{\mathcal{H}}_{\Omega},
$$

where $\hat{T}_{\rho}=\partial^{2} / \partial \rho^{2}+(5 / \rho) \partial / \partial \rho$ is the hyperradial kinetic energy operator and $\hat{\mathcal{H}}_{\Omega}$ is defined as

$$
\hat{\mathcal{H}}_{\Omega}=\frac{\hbar^{2}}{2 m} \frac{\hat{L}^{2}(\Omega)}{\rho^{2}}+\sum_{i<j} V(i, j),
$$

where $\hat{L}^{2}(\Omega)$ is the grand-angular operator, and $m$ is an arbitrary normalization mass. The operator $\hat{\mathcal{H}}_{\Omega}$ contains all the dependence on the hyperangles and the potential energy, which has been taken to include two-body forces only. Eventually three-body forces can be considered as well. This Hamiltonian can be solved for fixed values of $\rho$, such that the angular eigenfunctions $\Phi_{n}(\rho, \Omega)$ satisfy

$$
\hat{\mathcal{H}}_{\Omega} \Phi_{n}^{J M}(\rho, \Omega)=\frac{\hbar^{2}}{2 m} \frac{1}{\rho^{2}} \lambda_{n}(\rho) \Phi_{n}^{J M}(\rho, \Omega) .
$$

The set of angular eigenfunctions $\left\{\Phi_{n}^{J M}(\rho, \Omega)\right\}$ forms the HA basis with definite values of the total angular momentum and projection $J M$. They form a complete basis that can be used to expand the three-body wave function. The advantage of this basis is that the large distance behavior of each term can be related to the different open channels. In particular, the possible elastic, inelastic, and recombination $1+2$ channels are associated with specific adiabatic terms [6], whose corresponding eigenvalues $\lambda_{n}(\rho)$ go asymptotically as $2 m E_{d} \rho^{2} / \hbar^{2}$, where $E_{d}$ is now the binding energy of the dimer in that specific $1+2$ channel. The remaining infinitely many adiabatic basis terms describe three free particles in the continuum, and each of their corresponding eigenvalues $\lambda_{n}(\rho)$ behaves at large distances as $K(K+4)$, where $K$ is the grand-angular quantum number. Therefore, each breakup channel is associated with a single value of $K$. In other words, if a breakup angular eigenfunction $\Phi_{n}^{J M}(\rho, \Omega)$ is expanded in terms of the HH basis, at very large distances, only the $\mathrm{HH}$ basis elements with that specific value of $K$ survive. Asymptotically the HA basis describing the breakup channels coincides with the HH basis since, in this case, the HA basis elements are eigenfunctions of the $\hat{\mathcal{H}}_{\Omega}$ operator given in Eq. (18) without the interaction term.

In the following we shall focus on a $1+2$ process where inelastic or recombination channels are not possible. This means that there is only one possible dimer in the $1+2$ system, which can be either the target or the projectile, not having any bound excited state. The inclusion of additional $1+2$ channels does not present any intrinsic difficulties [4], and they are not relevant for the description of the breakup channel discussed in this work. In fact, the $n-d$ reaction that we consider corresponds precisely to this kind of reaction (the deuteron does not have excited states and is the only two-nucleon bound system).

In Appendix D we show that the adiabatic expansion of the outgoing three-body wave function describing the breakup of the dimer can be written as [Eq. (D13)]

$$
\begin{aligned}
\Psi_{\sigma_{d} \sigma_{p}}^{\sigma_{i} \sigma_{j} \sigma_{k}}= & (2 \pi)^{3 / 2} \sum_{J M} \sum_{n>1} \frac{1}{(\kappa \rho)^{5 / 2}} f_{n 1}^{J}(\rho) \\
& \times\left\langle\sigma_{i} \sigma_{j} \sigma_{j} \mid \Phi_{n}^{J M}\left(\rho, \Omega_{\rho}\right)\right\rangle\left\langle\sigma_{d} \sigma_{p}\left|\Phi_{1}^{J M}\left(\kappa, \Omega_{\kappa}\right)\right|^{*},\right.
\end{aligned}
$$

where $\Phi_{1}^{J M}\left(\kappa, \Omega_{\kappa}\right)$ is the adiabatic angular function (in momentum space) associated with the incident $1+2$ channel 
(channel 1), and $J$ and $M$ are the total angular momentum and projection of the three-body (projectile + target) system. The summation over $n$ refers to all the breakup adiabatic channels, whose corresponding angular functions are given by $\Phi_{n}^{J M}\left(\rho, \Omega_{\rho}\right)$ (as mentioned above, we have assumed that the incident channel 1 is the only $1+2$ channel in the three-body system). The wave function is projected over the initial spin states $\left|\sigma_{d} \sigma_{p}\right\rangle$ of target and projectile and the final spin states $\left|\sigma_{i} \sigma_{j} \sigma_{k}\right\rangle$ of the three particles after the breakup.

The hyperradial functions $f_{n 1}^{J}(\rho)$ in Eq. (20) depend on the total angular momentum $J$ and are obtained by solving the set of coupled differential equations

$$
\begin{aligned}
& {\left[-\frac{d^{2}}{d \rho^{2}}+\frac{\lambda_{n}(\rho)+\frac{15}{4}}{\rho^{2}}-\frac{2 m E}{\hbar^{2}}\right] f_{n 1}^{J}(\rho)} \\
& -\sum_{n}\left(2 P_{n n^{\prime}}(\rho) \frac{d}{d \rho}+Q_{n n^{\prime}}(\rho)\right) f_{n^{\prime} 1}^{J}(\rho)=0
\end{aligned}
$$

where the eigenvalues $\lambda_{n}(\rho)$ in Eq. (19) enter as effective potentials and where the functions of the hyperradius $P_{n n^{\prime}}$ and $Q_{n n^{\prime}}$ couple the different adiabatic terms. Details are given in [4] and [6].

For the breakup channels $(n>1)$ the radial wave functions $f_{n 1}^{J}(\rho)$ behave asymptotically as [19]

$$
f_{n 1}^{J}(\rho) \rightarrow \frac{\sqrt{\kappa \rho}}{2} \mathcal{S}_{1 n}^{J} H_{K+2}^{(1)}(\kappa \rho) \rightarrow \frac{1}{2} i^{-K} \mathcal{S}_{1 n}^{J} \sqrt{\frac{2}{\pi}} e^{i \frac{3 \pi}{4}} e^{i \kappa \rho},
$$

where $K$ is the grand-angular quantum number associated with the breakup adiabatic channel $n, H_{K+2}^{(1)}$ is a Hankel function of first kind, and $\mathcal{S}_{1 n}^{J}$ is the corresponding matrix element of the $\mathcal{S}$-matrix. Using the expression above, we can write the asymptotic behavior of the outgoing wave function in Eq. (20) as

$$
\Psi_{\sigma_{d} \sigma_{n}}^{\sigma_{1} \sigma_{2} \sigma_{3}} \rightarrow \frac{e^{i \kappa \rho}}{\rho^{5 / 2}} A_{\sigma_{d} \sigma_{n}}^{\sigma_{1} \sigma_{2} \sigma_{3}}
$$

where the breakup transition amplitude is given by

$$
\begin{aligned}
A_{\sigma_{d} \sigma_{p}}^{\sigma_{i} \sigma_{j} \sigma_{k}}= & \frac{2 \pi}{\kappa^{5 / 2}} e^{i \frac{3 \pi}{4}} \sum_{J M} \sum_{n>1} i^{-K} \mathcal{S}_{1 n}^{J}\left\langle\sigma_{i} \sigma_{j} \sigma_{k} \mid \Phi_{n}^{J M}\left(\Omega_{\rho}\right)\right\rangle \\
& \times\left\langle\sigma_{d} \sigma_{p} \mid \Phi_{1}^{J M}\left(\kappa, \Omega_{\kappa}\right)\right\rangle^{*}
\end{aligned}
$$

and $\Phi_{n}^{J M}\left(\Omega_{\rho}\right)=\lim _{\rho \rightarrow \infty} \Phi_{n}^{J M}\left(\rho, \Omega_{\rho}\right)$.

The asymptotic behavior of the angular eigenfunction $\Phi_{1}^{J M}\left(\kappa, \Omega_{\kappa}\right)$ is derived in Appendix $\mathrm{E}$ and, for the particular case of relative $s$ waves between the particles, takes the form [Eq. (E10)]

$$
\begin{aligned}
& \left\langle\sigma_{d} \sigma_{p} \mid \Phi_{1}^{J M}\left(\kappa, \Omega_{\kappa}\right)\right\rangle^{*} \\
& \rightarrow \frac{1}{\sqrt{4 \pi}}\left(\frac{\mu_{x}}{m}\right)^{3 / 4} \frac{\kappa^{2}}{\sqrt{k_{y}^{(\mathrm{in})}}}\left\langle s_{d} \sigma_{d} s_{p} \sigma_{p} \mid J M\right\rangle,
\end{aligned}
$$

where $k_{y}^{(\mathrm{in})}$ is related to the incident relative projectile-dimer momentum $p_{y}^{(\text {in) }}$ through Eq. (6) and $\mu_{x}$ is the reduced mass of the two particles in the dimer. The expression above permits us to write the transition amplitude given in Eq. (24) as

$$
\begin{aligned}
A_{\sigma_{d} \sigma_{n}}^{\sigma_{1} \sigma_{2} \sigma_{3}}= & \frac{\sqrt{\pi}}{\sqrt{\kappa k_{y}^{(\mathrm{in})}}}\left(\frac{\mu_{x}}{m}\right)^{3 / 4} e^{i \frac{3 \pi}{4}} \sum_{J M}\left\langle s_{d} \sigma_{d} s_{p} \sigma_{p} \mid J M\right\rangle \\
& \times \sum_{n>1} i^{-K} \mathcal{S}_{1 n}^{J}\left\langle\sigma_{1} \sigma_{2} \sigma_{3} \mid \Phi_{n}^{J M}\left(\Omega_{\rho}\right)\right\rangle,
\end{aligned}
$$

which is valid for relative $s$ waves only.

The angular eigenfunctions $\Phi_{n}^{J M}\left(\rho, \Omega_{\rho}\right)$ can be decomposed in the three Faddeev amplitudes as (see Refs. [1] and [2] for details)

$$
\begin{aligned}
\Phi_{n}^{J M}(\rho, \Omega)= & \Phi_{n}^{(i) J M}\left(\rho, \Omega_{i}\right)+\Phi_{n}^{(j) J M}\left(\rho, \Omega_{j}\right) \\
& +\Phi_{n}^{(k) J M}\left(\rho, \Omega_{k}\right)
\end{aligned}
$$

where each of the three components of the angular eigenfunction is written in terms of each of the three possible sets of Jacobi coordinates. Moreover, in the present work the angular eigenfunctions $\Phi_{n}^{J M}\left(\rho, \Omega_{i}\right)$ are expanded in terms of the $\mathrm{HH}$ basis. In general, the corresponding expansion coefficients contain the dependence of the angular eigenfunction on $\rho$. Asymptotically each breakup adiabatic channel is associated with some specific value of $K$, and the eigenfunction becomes a $\rho$-independent expansion. More precisely, it results in a linear combination of $\mathrm{HH}$ functions having a well-defined grand-angular quantum number. In particular, if we consider only relative $s$ waves between the particles, the following expression can be obtained:

$$
\begin{aligned}
& \left\langle\sigma_{i} \sigma_{j} \sigma_{k} \mid \Phi_{n}^{(i) J M}\left(\Omega_{i}\right)\right\rangle \\
& =\frac{1}{4 \pi} \sum_{s_{x_{i}}} C_{K s_{x_{i}}}^{(n)} N_{K} P_{\nu}^{\left(\frac{1}{2} \frac{1}{2}\right)}\left(\cos 2 \alpha_{i}\right)\left\langle\sigma_{i} \sigma_{j} \sigma_{k} \mid \chi_{s_{x_{i}} s_{y_{i}}}^{J M}\right\rangle,
\end{aligned}
$$

where the $C$ s are the coefficients in the expansion, $N_{K}$ is a normalization coefficient, and $P_{\nu}^{\left(\frac{1}{2} \frac{1}{2}\right)}$ is a Jacobi polynomial. Moreover, $K$ is associated with the asymptotic behavior of the adiabatic channel $v=K / 2, s_{x}$ is the coupling between the spins of the two particles used to construct the $\boldsymbol{x}$ Jacobi coordinate, $s_{y}$ is the spin of the third particle, and $\chi_{s_{x} s_{y}}^{J M}$ is the total three-body spin function arising from the coupling of $s_{x}$ and $s_{y}$ to the total angular momentum $J$ with projection $M$. Taking this into account, the transition amplitude in Eq. (26) can be written in a more compact form as

$$
\begin{aligned}
A_{\sigma_{d} \sigma_{p}}^{\sigma_{i} \sigma_{j} \sigma_{k}}= & \sum_{J M}\left\langle s_{d} \sigma_{d} s_{p} \sigma_{p} \mid J M\right\rangle \\
& \times \sum_{q=1}^{3} \sum_{s_{x_{q}}} A_{s_{x_{q}}}(q)\left\langle\sigma_{i} \sigma_{j} \sigma_{k} \mid \chi_{s_{x_{q}} s_{y q}}^{J M}\right\rangle,
\end{aligned}
$$

where the index $q$ numbers the three possible sets of Jacobi coordinates and

$$
\begin{aligned}
A_{s_{x_{q}}}(q)= & \frac{1}{4 \sqrt{\pi}} \frac{1}{\sqrt{\kappa k_{y}^{(\mathrm{in})}}}\left(\frac{\mu_{x}}{m}\right)^{3 / 4} e^{i \frac{3 \pi}{4}} \\
& \times \sum_{n>1} i^{-K} C_{K s_{x_{q}}}^{(n)} \mathcal{S}_{1 n}^{J} N_{K} P_{v}^{\left(\frac{1}{2} \frac{1}{2}\right)}\left(\cos 2 \alpha_{q}\right),
\end{aligned}
$$


where we have made use of Eq. (28). From Eq. (29) we can then finally write

$$
\begin{aligned}
& \sum_{\sigma_{p} \sigma_{d}} \sum_{\sigma_{i} \sigma_{j} \sigma_{k}}\left|A_{\sigma_{d} \sigma_{p}}^{\sigma_{i} \sigma_{j} \sigma_{k}}\right|^{2} \\
& =\sum_{J}(2 J+1)\left\{\sum_{p, q=1}^{3} \sum_{s_{x_{p}} s_{x_{q}}} A_{s_{x_{p}}}^{*}(p) A_{s_{x_{q}}}(q)\left\langle\chi_{s_{x_{p}} s_{y_{p}}}^{J} \mid \chi_{s_{x_{q}} s_{y_{q}}}^{J}\right\rangle\right\},
\end{aligned}
$$

where $p$ and $q$ run over the three possible sets of Jacobi coordinates, and $\left|\chi_{s_{x p} s_{y p}}^{J}\right\rangle$ is the three-body spin function in the Jacobi set $p$, where the spin $s_{x_{p}}$, associated with the Jacobi coordinate $\boldsymbol{x}_{p}$, couples to the spin $s_{y_{p}}$ of the third particle to give the total three-body angular momentum $J$ (all the orbital angular momenta are assumed to be 0). Finally, Eq. (31), together with Eq. (30), permits us to obtain the cross section in the center-of-mass frame in Eq. (11) and, therefore, the cross section in the laboratory frame as given by Eq. (14).

It should be noted that the transition amplitude in Eq. (26) is obtained from the asymptotic behavior of the wave function in Eq. (20). This means that the hyperangles $\Omega_{\rho}$ entering into Eq. (26) or (30) are the asymptotic hyperangles, which are known to be the same in coordinate and momentum space.

\section{Integral relation for the breakup transition amplitude}

The working equation in the calculation of the breakup amplitude using the HA basis is Eq. (30). The feasibility of the method could be limited by the number of adiabatic terms needed in the expansion, given by the index $n$, which, in principle, runs up to $\infty$. Since asymptotically the HA and the $\mathrm{HH}$ bases tend to be the same, the coefficients $C_{K s_{x_{q}}}^{(n)}$ have the property of being close to a non-zero constant when $K$ takes the value associated with the asymptotic behavior of the adiabatic potential $n$ and close to 0 otherwise. Therefore the number of terms in the expansion is intrinsically related to the ability of the $\mathrm{HH}$ basis to describe the asymptotic configurations.

If the hyperangle $\alpha_{q}=\arctan \left(k_{x_{q}} / k_{y_{q}}\right)$ approaches 0 , the relative momentum of the two particles connected by the $\boldsymbol{x}_{q}$ Jacobi coordinate approaches 0 as well, and the two particles appear in an almost-zero-energy relative state. As mentioned before, this produces a kind of clusterization in the breakup amplitude very difficult to describe with the HH basis. In fact, for this particular geometry, the breakup adiabatic angular eigenfunctions entering into the transition amplitude [see Eq. (26)] should be similar to the one corresponding to a $1+2$ channel, given by Eq. (E1), but replacing the bound dimer wave function with the zero-energy two-body wave function.

To illustrate this point we take the $n-d$ case with total spin $J=3 / 2$ as an example. In this case, according to Eq. (E1), for geometries having $\alpha \approx 0(x \ll y)$ and assuming relative $s$ waves between the particles, we should get

$$
\Phi_{n}^{J=3 / 2}\left(\rho, \Omega_{\rho}\right) \stackrel{\rho \rightarrow \infty}{\rightarrow} \frac{u^{s_{x}=1}}{r_{n p}}
$$

for all the angular eigenfunctions associated with breakup channels $(n>1)$, where $u^{s_{x}=1} / r_{n p}$ is the zero-energy neutron-

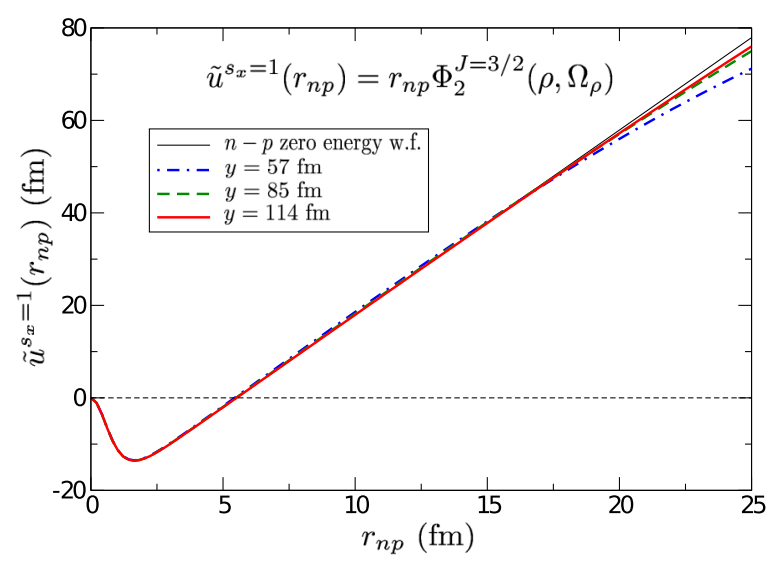

FIG. 1. (Color online) Wave function $\tilde{u}^{s_{x}=1}\left(r_{n p}\right)$ obtained from the lowest breakup angular eigenfunction $\Phi_{2}^{J=1 / 2}\left(\rho, \Omega_{\rho}\right)$ for the $n-d$ case with total angular momentum $J=3 / 2$ (see text) and for three fixed values of the Jacobi coordinate $y: 57 \mathrm{fm}$ (dot-dashed line), $85 \mathrm{fm}$ (dashed line), and $114 \mathrm{fm}$ (solid line). The thin solid line is the zero-energy neutron-proton wave function $u^{s_{x}=1}$ introduced in Eq. (32).

proton wave function, and $r_{n p}$ is the relative distance between the neutron and the proton. In Fig. 1 we plot the function $\tilde{u}^{s_{x}=1}\left(r_{n p}\right)=r_{n p} \Phi_{2}^{J=3 / 2}\left(\rho, \Omega_{\rho}\right)$ as a function $r_{n p}$ for three fixed values of the Jacobi coordinate $y: 57 \mathrm{fm}$ (dot-dashed curve), $85 \mathrm{fm}$ (dashed curve), and $114 \mathrm{fm}$ (solid curve). Obviously, the larger the value of $y$, the smaller the value of the hyperangle $\alpha$ associated with a given $r_{n p}$, and therefore the more closely the function $\tilde{u}^{s_{x}=1}$ should approach the zero-energy two-body function $u^{s_{x}=1}$ given in Eq. (32), which is shown in Fig. 1 by the thin solid line. The expected behavior is what we observe in the figure, where, as we can see, for $y=114 \mathrm{fm}$ the function $\tilde{u}^{s_{x}=1}$ (thick solid curve) matches the two-body wave function $u^{s_{x}=1}$ (thin solid curve) pretty well up to almost $20 \mathrm{fm}$ (the thin solid curve has been scaled to fit the same minimum as $\tilde{u}^{s_{x}=1}$ ).

The zero-energy two-body wave function is linear in the relative radial coordinate (see Fig. 1) and, therefore, proportional to $\sin \alpha_{q}$. The reconstruction of this behavior by use of an expansion in terms of $\cos 2 \alpha_{q}$, as in the expansion given in Eq. (30), requires, in principle, infinitely many terms. Accordingly, as $\alpha_{q} \rightarrow 0$ the number of adiabatic terms needed to get a convergent value for $A_{s_{x p}}(p)$ increases without limit. Hence, Eq. (30) is not operative in this particular situation.

To overcome this problem we develop in this section an alternative expression for the transition amplitude where the adiabatic expansion in Eq. (26), in terms of the coefficients $C_{K s_{x q}}^{(n)}$, does not enter explicitly. This procedure is more expensive from a numerical point of view but more accurate in the kinematic regions where $\alpha_{\kappa} \approx 0$. The starting point here is the well-known expansion of the three-body plane wave in terms of the HHs:

$$
\begin{aligned}
e^{i\left(\boldsymbol{k}_{x} \cdot \boldsymbol{x}+\boldsymbol{k}_{y} \cdot \boldsymbol{y}\right)}\left|\sigma_{i} \sigma_{j} \sigma_{k}\right\rangle= & \frac{(2 \pi)^{3}}{(\kappa \rho)^{2}} \sum_{J M} \sum_{[K]} i^{K} J_{K+2}(\kappa \rho) \mathcal{Y}_{[K]}^{J M}\left(\Omega_{\rho}\right) \\
& \times\left\langle\sigma_{i} \sigma_{j} \sigma_{k}\right| \mathcal{Y}_{[K]}^{J M *}\left(\Omega_{\kappa}\right),
\end{aligned}
$$


where $\mathcal{Y}_{[K]}^{J M}$ is an $\mathrm{HH}$ function coupled to a three-body spin function [1]. All the quantum numbers are collected into the set $[K] \equiv\left\{K, \ell_{x}, \ell_{y}, L, s_{x}, S\right\}$. On the other hand, the angular eigenfunctions in the adiabatic expansion corresponding to breakup channels $(n>1)$ are, asymptotically, a linear combination of HHs. The two bases can be formally related as

$$
\mathcal{Y}_{[K]}^{J M}(\Omega)=\sum_{n>1}\left\langle\Phi_{n}^{J M}(\Omega)\left|\mathcal{Y}_{[K]}^{J M}(\Omega)\right| \Phi_{n}^{J M}(\Omega),\right.
$$

with the sum over $n$ restricted to those channels associated with the grand-angular quantum number $K$. Replacing in Eq. (33) we obtain

$$
\begin{aligned}
e^{i\left(\boldsymbol{k}_{x} \cdot \boldsymbol{x}+\boldsymbol{k}_{y} \cdot \boldsymbol{y}\right)}\left|\sigma_{i} \sigma_{j} \sigma_{k}\right\rangle= & \frac{(2 \pi)^{3}}{(\kappa \rho)^{2}} \sum_{J M} \sum_{n>1} i^{K} J_{K+2}(\kappa \rho) \Phi_{n}^{J M}\left(\Omega_{\rho}\right) \\
& \times\left\langle\sigma_{i} \sigma_{j} \sigma_{k}\left|\Phi_{n}^{J M}\left(\Omega_{\kappa}\right)\right|^{*}\right.
\end{aligned}
$$

where we have used that $\sum_{[K]}\left\langle\Phi_{n}^{J M} \mid \mathcal{Y}_{[K]}^{J M}\right\rangle\left\langle\mathcal{Y}_{[K]}^{J M} \mid \Phi_{n^{\prime}}^{J M}\right\rangle=$ $\delta_{n n^{\prime}}$.

As shown in Ref. [6], the regular outgoing wave functions for the breakup channels are given by

$$
F_{n}=\sqrt{\frac{\pi}{2}} \frac{1}{\rho^{2}} J_{K+2}(\kappa \rho) \Phi_{n}^{J M}\left(\Omega_{\rho}\right) .
$$

Therefore Eq. (35) can be written in terms of $F_{n}$, and in particular, it can be used to write the matrix element

$$
\begin{aligned}
& \left.\left\langle\Psi_{1}^{J M}|\hat{\mathcal{H}}-E| e^{i\left(\boldsymbol{k}_{x} \cdot \boldsymbol{x}+\boldsymbol{k}_{y} \cdot \boldsymbol{y}\right)} \mid \sigma_{i} \sigma_{j} \sigma_{k}\right\rangle\right\rangle \\
& =\frac{(2 \pi)^{3}}{\kappa^{2}} \sqrt{\frac{2}{\pi}} \sum_{n>1} i^{K}\left\langle\Psi_{1}^{J M}|\hat{\mathcal{H}}-E| F_{n}\right\rangle\left\langle\sigma_{i} \sigma_{j} \sigma_{k} \mid \Phi_{n}^{J M}\left(\Omega_{\kappa}\right)\right\rangle^{*},
\end{aligned}
$$

where $\hat{\mathcal{H}}$ is the three-body Hamiltonian, $E$ is the total three-body energy, and $\Psi_{1}^{J M}$ is the three-body wave function corresponding to the incoming $1+2$ channel labeled 1 .

In Ref. [6] it was also shown that the $\mathcal{K}$ matrix for a breakup process can be obtained through two integral relations that provide the two matrices, $A$ and $B$, such that the $\mathcal{K}$ matrix of the reaction takes the form $\mathcal{K}=-A^{-1} B$. In particular, the $i j$ term of each of these two matrices is given by

$$
\begin{aligned}
A_{i j} & =-\frac{2 m}{\hbar^{2}}\left\langle\Psi_{i}^{J M}|\hat{\mathcal{H}}-E| G_{j}\right\rangle, \\
B_{i j} & =\frac{2 m}{\hbar^{2}}\left\langle\Psi_{i}^{J M}|\hat{\mathcal{H}}-E| F_{j}\right\rangle,
\end{aligned}
$$

where $G_{j}$ is defined as $F_{j}$, Eq. (36), but replacing the regular Bessel function $J_{K+2}$ with the irregular Bessel function $Y_{K+2}$.

Use of Eq. (39) permits us to write Eq. (37) as

$$
\begin{aligned}
& \left.\left\langle\Psi_{1}^{J M}|\hat{\mathcal{H}}-E| e^{i\left(\boldsymbol{k}_{x} \cdot \boldsymbol{x}+\boldsymbol{k}_{y} \cdot \boldsymbol{y}\right)} \mid \sigma_{i} \sigma_{j} \sigma_{k}\right\rangle\right\rangle \\
& =\frac{(2 \pi)^{3}}{\kappa^{2}} \sqrt{\frac{2}{\pi}} \frac{\hbar^{2}}{2 m} \sum_{n>1} i^{K} B_{1 n}\left\langle\sigma_{i} \sigma_{j} \sigma_{k}\left|\Phi_{n}^{J M}\left(\Omega_{\kappa}\right)\right|^{*},\right.
\end{aligned}
$$

where it is important to keep in mind that 1 refers to the incoming channel $(1+2$ channel $)$ and $n$ refers to the breakup channels (therefore $n>1$ ).
Note that the matrix element in Eq. (40) is just the first component of a column vector whose $i$ th term is given by $\left\langle\Psi_{i}^{J M}|\hat{\mathcal{H}}-E|\right.$ planewave $\rangle(i=1, \ldots, N$, where $N$ is the number of adiabatic channels included in the calculation), where for $i>2$ the wave functions $\Psi_{i}^{J M}$ describe scattering processes with three ingoing particles. If we multiply from the left such a column vector by any $N \times N$ matrix $M$, the result would be a new column vector whose first term would be given by Eq. (40) but replacing $B_{1 n}$ with $(M \cdot B)_{1 n}$. Therefore, if we take $M=A^{-1}$, the first component of the new vector will be given by Eq. (40) but replacing $B_{1 n}$ with $\left(A^{-1} B\right)_{1 n}$, which is nothing but $-\mathcal{K}_{1 n}$. For the same reason, since $\mathcal{K}=-i(\mathcal{S}+\mathbb{I})^{-1}(\mathcal{S}-\mathbb{I})$, if we multiply from the left the new column vector by $(\mathcal{S}+\mathbb{I})$, the new matrix element in Eq. (40) would be given not in terms of $\mathcal{K}_{1 n}$, but in terms of $(\mathcal{S}-\mathbb{I})_{1 n}$, which reduces to $\mathcal{S}_{1 n}$ in our case, where 1 refers to the $1+2$ incident channel and $n$ corresponds to outgoing breakup channels $(n>1)$.

Summarizing, the first step is to compute the $A$ and $B$ matrices as described in Ref. [6], from which the $\mathcal{S}$ matrix of the reaction can be obtained. Successively a proper normalized scattering state is constructed according to

$$
\Psi^{J M} \rightarrow(\mathcal{S}+\mathbb{I}) A^{-1} \Psi^{J M}
$$

and once this is done the matrix element given in Eq. (40) transforms into

$$
\begin{aligned}
& \left.\left\langle\Psi_{1}^{J M}|\hat{\mathcal{H}}-E| e^{i\left(\boldsymbol{k}_{x} \cdot \boldsymbol{x}+\boldsymbol{k}_{y} \cdot \boldsymbol{y}\right)} \mid \sigma_{i} \sigma_{j} \sigma_{k}\right\rangle\right\rangle \\
& =i \frac{(2 \pi)^{3}}{\kappa^{2}} \sqrt{\frac{2}{\pi}} \frac{\hbar^{2}}{2 m} \sum_{n>1} i^{-K} \mathcal{S}_{1 n}\left\langle\sigma_{i} \sigma_{j} \sigma_{k}\left|\Phi_{n}^{J M}\left(\Omega_{\kappa}\right)\right|^{*},\right.
\end{aligned}
$$

where the replacement of $i^{K}$ with $i^{-K}$ is irrelevant, since the difference is a factor $i^{2 K}=(-1)^{K}$, which is either 1 for positive-parity states ( $K$ even for all $n$ ) or -1 for negativeparity states ( $K$ odd for all $n$ ). In any case, this does not play any role, since the calculation of the cross section will contain the square of the matrix element above.

In Eq. (42) the angular function $\left\langle\sigma_{i} \sigma_{j} \sigma_{k} \mid \Phi_{n}\left(\Omega_{\kappa}\right)\right\rangle$ is just the Fourier transform of Eq. (28), whose analytical form is exactly the same as in Eq. (28) but with the angles understood in momentum space (asymptotically the hyperangles in coordinate and momentum space are the same).

Comparing Eqs. (26) and (42), we can identify the breakup transition amplitude as

$$
\begin{aligned}
A_{\sigma_{d} \sigma_{n}}^{\sigma_{i} \sigma_{j} \sigma_{k}}= & \frac{\pi}{\sqrt{2}} \frac{\kappa^{2}}{\sqrt{\kappa k_{y}}} \frac{1}{(2 \pi)^{3}}\left(\frac{\mu_{x}}{m}\right)^{3 / 4} e^{i \frac{\pi}{4}} \frac{2 m}{\hbar^{2}} \\
& \times \sum_{J M}\left\langle s_{d} \sigma_{d} s_{p} \sigma_{p} \mid J M\right\rangle\left\langle\Psi_{1}^{J M}\right| \hat{\mathcal{H}} \\
& \left.\left.-E\left|e^{i\left(\boldsymbol{k}_{x} \cdot \boldsymbol{x}+\boldsymbol{k}_{y} \cdot \boldsymbol{y}\right)}\right| \sigma_{i} \sigma_{j} \sigma_{k}\right\rangle\right\rangle .
\end{aligned}
$$

Since the plane wave is the solution of the free Hamiltonian, the matrix element in Eq. (42) is actually the sum of three matrix elements, each of them involving one of the three two-body potentials. In turn, each of these three matrix elements is more easily treated in the Jacobi set such that the potential depends 
on the $\boldsymbol{x}$ coordinate only. In other words, we can write

$$
\begin{aligned}
& \left.\left\langle\Psi_{1}^{J M}|\hat{\mathcal{H}}-E| e^{i\left(\boldsymbol{k}_{x} \cdot \boldsymbol{x}+\boldsymbol{k}_{y} \cdot \boldsymbol{y}\right)} \mid \sigma_{i} \sigma_{j} \sigma_{k}\right\rangle\right\rangle \\
& \left.=\sum_{q=1}^{3}\left\langle\Psi_{1}^{J M}\left|V_{q}\left(x_{q}\right)\right| e^{i\left(\boldsymbol{k}_{x_{q}} \cdot \boldsymbol{x}_{q}+\boldsymbol{k}_{y_{q}} \cdot \boldsymbol{y}_{q}\right)} \mid \sigma_{i} \sigma_{j} \sigma_{k}\right\rangle\right\rangle,
\end{aligned}
$$

where $V_{q}\left(x_{q}\right)$ is the two-body potential between the two particles connected by the Jacobi coordinate $\boldsymbol{x}_{q}$.

If we now consider that

$$
\begin{aligned}
& \left.\left\langle\Psi_{1}^{J M}\left|V_{q}\left(x_{q}\right)\right| e^{i\left(\boldsymbol{k}_{x_{q}} \cdot \boldsymbol{x}_{q}+\boldsymbol{k}_{y_{q}} \cdot \boldsymbol{y}_{q}\right)} \mid \sigma_{i} \sigma_{j} \sigma_{k}\right\rangle\right\rangle \\
& \left.=\sum_{s_{x_{q}}}\left\langle\Psi_{1}^{J M}\left|V_{q}\left(x_{q}\right)\right| e^{i\left(\boldsymbol{k}_{x_{q}} \cdot \boldsymbol{x}_{q}+\boldsymbol{k}_{y_{q}} \cdot \boldsymbol{y}_{q}\right)} \mid \chi_{s_{x_{q}} s_{y q}}^{J M}\right\rangle\right\rangle \\
& \quad \times\left\langle\chi_{s_{x_{q}} s_{y_{q}}}^{J M} \mid \sigma_{i} \sigma_{j} \sigma_{k}\right\rangle,
\end{aligned}
$$

where $s_{x_{q}}$ is the spin of the two-body system connected by the $\boldsymbol{x}_{q}$ Jacobi coordinate, we can then, by use of Eqs. (44) and (45), write the transition amplitude in Eq. (44) exactly as given in Eq. (29), where now

$$
\begin{aligned}
A_{s_{x_{q}}}(q)= & \frac{\pi}{\sqrt{2}} \frac{\kappa^{2}}{\sqrt{\kappa k_{y_{q}}}} \frac{1}{(2 \pi)^{3}}\left(\frac{\mu_{x}}{m}\right)^{3 / 4} e^{i \frac{\pi}{4}} \\
& \left.\times \frac{2 m}{\hbar^{2}}\left\langle\Psi_{1}^{J M}\left|V_{q}\left(x_{q}\right)\right| e^{i\left(\boldsymbol{k}_{x_{q}} \cdot \boldsymbol{x}_{q}+\boldsymbol{k}_{y_{q}} \cdot \boldsymbol{y}_{q}\right)} \mid \chi_{s_{x_{q}} s_{y_{q}}}^{J}\right\rangle\right) .
\end{aligned}
$$

Thus, Eq. (46) permits us to obtain Eq. (31), and therefore the cross sections in Eqs. (11) and (14). Contrary to what happens in Eq. (30), Eq. (46) does not contain any expansion of the outgoing wave, and in principle, the infinitely many breakup adiabatic terms are included. A discussion of the calculation of the matrix element contained in Eq. (46) is given in the next section.

\section{CALCULATION OF THE BREAKUP INTEGRAL RELATION USING THE HA FORMALISM}

Let us denote the matrix element to be computed as

$$
\left.M_{s_{x_{i}}}\left(\boldsymbol{k}_{x_{i}}, \boldsymbol{k}_{y_{i}}\right)=\left\langle\Psi_{1}^{J M}\left|V_{i}\left(x_{i}\right)\right| e^{i\left(\boldsymbol{k}_{x_{i}} \cdot \boldsymbol{x}_{i}+\boldsymbol{k}_{y_{i}} \cdot \boldsymbol{y}_{i}\right)} \mid \chi_{s_{x_{i}} s_{y_{i}}}^{J M}\right\rangle\right\rangle \text {. }
$$

The three-body wave function $\Psi_{1}^{J M}$ is expanded in terms of the adiabatic angular eigenfunctions, which in turn are expanded in terms of the HHs (see also Refs. [4] and [6]):

$$
\Psi_{1}^{J M}\left(\boldsymbol{x}_{i}, \boldsymbol{y}_{i}\right)=\frac{1}{\rho^{5 / 2}} \sum_{n} f_{n 1}(\rho) \sum_{[K]} C_{[K]}^{(n)}(\rho) \mathcal{Y}_{[K]}^{J M}\left(\Omega_{i}\right),
$$

where the coefficients $C_{[K]}^{(n)}$ reduce to $C_{K, s_{x_{i}}}^{(n)}$ in the case of $s$ waves and where we have selected the $i$ arrangement of the Jacobi coordinates to construct the three-body $\mathrm{HH}$-spin functions $\mathcal{Y}_{[K]}^{J M}$. It should be noted that it is convenient to expand the three-body wave function $\Psi_{1}^{J M}$ in the set $i$ of Jacobi coordinates in which the coordinate $\boldsymbol{x}_{i}$ is the same appearing in the interaction potential, as given in Eq. (47). Therefore for each of the three integrals given in Eq. (44), the corresponding set of Jacobi coordinates is used. Inserting the above expansion into Eq. (47), we get that the matrix element takes the form (the index $i$ is omitted henceforth and we consider $s$ waves only)

$$
\begin{aligned}
M_{s_{x}}\left(\boldsymbol{k}_{x}, \boldsymbol{k}_{y}\right)= & \frac{1}{4 \pi} \int d^{3} x d^{3} y \frac{1}{\rho^{5 / 2}} \sum_{n} f_{n 1}(\rho) \\
& \times \sum_{K} C_{K, s_{x}}^{(n)}(\rho) N_{K} P_{v}^{\left(\frac{1}{2}, \frac{1}{2}\right)} \\
& \times(\cos 2 \alpha)\left\langle\chi_{s_{x} s_{y}}^{J M}|V(x)| \chi_{s_{x} s_{y}}^{J M}\right\rangle e^{i\left(\boldsymbol{k}_{x_{i}} \cdot \boldsymbol{x}_{i}+\boldsymbol{k}_{y_{i}} \cdot \boldsymbol{y}_{i}\right)},
\end{aligned}
$$

where the two-body potential is assumed not to mix different $s_{x}$ values.

Keeping in mind that the input in our calculation will be the directions of the momenta of two of the outgoing particles and that, as already shown, for each value of the arclength $S$ it is possible to construct the full momenta of the three particles after the breakup, these three momenta permit us to construct $\boldsymbol{k}_{x}$ and $\boldsymbol{k}_{y}$, and the matrix element in Eq. (49) can then be computed as a function of the arclength $S$. Note also that in Eq. (49), since only $s$ waves are assumed to contribute, the full dependence of the integrand on $\Omega_{x}$ and $\Omega_{y}$ is contained in the exponential. The integration over $\Omega_{x}$ and $\Omega_{y}$ can then be made analytically, leading to the expression

$$
\begin{aligned}
& \int d \Omega_{x} d \Omega_{y}\left(\cos \left(\boldsymbol{k}_{x} \cdot \boldsymbol{x}+\boldsymbol{k}_{y} \cdot \boldsymbol{y}\right)+i \sin \left(\boldsymbol{k}_{x} \cdot \boldsymbol{x}+\boldsymbol{k}_{y} \cdot \boldsymbol{y}\right)\right) \\
& =(2 \pi)^{2} \frac{4 \sin \left(k_{x} x\right) \sin \left(k_{y} y\right)}{k_{x} x k_{y} y}
\end{aligned}
$$

which is real (the integral involving the sinus is just 0).

The remaining integral over $x$ and $y$ has to be performed numerically:

$$
\begin{aligned}
M_{s_{x}}\left(k_{x}, k_{y}\right)= & 4 \pi \int d x d y \frac{x y}{\rho^{5 / 2}} \sum_{n} f_{n 1}(\rho) \\
& \times \sum_{K} C_{K, s_{x}}^{(n)}(\rho) N_{K} P_{v}^{\left(\frac{1}{2}, \frac{1}{2}\right)}(\cos 2 \alpha) \\
& \times\left\langle\chi_{s_{x} s_{y}}^{J M}|V(x)| \chi_{s_{x} s_{y}}^{J M}\right\rangle \frac{\sin \left(k_{x} x\right) \sin \left(k_{y} y\right)}{k_{x} k_{y}} .
\end{aligned}
$$

As we can see, the dependence on the directions of $\boldsymbol{k}_{x}$ and $\boldsymbol{k}_{y}$ has disappeared. The dependence on the momenta is only through $k_{x}=\kappa \sin \alpha_{\kappa}$ and $k_{y}=\kappa \cos \alpha_{\kappa}$, where $\kappa=\sqrt{2 m E / \hbar^{2}}$ and where $E$ is the three-body energy above threshold. It is important not to get confused with $\alpha(=\arctan x / y)$, which is a variable in coordinate space to be integrated away, and $\alpha_{\kappa}\left(=\arctan k_{x} / k_{y}\right)$, which is a variable in momentum space and which takes a well-defined value for each value of the arclength $S$.

The integral over $x$ is limited by the short-range character of the potential $V(x)$. Therefore the numerical computation of the integral does not present any particular difficulties. In Fig. 2(a) we show two examples of the typical behavior of the integrand as a function of $x$ for two arbitrary fixed values of $y$. The curves correspond to the $n$ - $d$ breakup reaction, whose details are given later. They have been obtained for two fixed values of the $y$ coordinate. As we can see, the function becomes basically 0 for rather small values of $x$. 

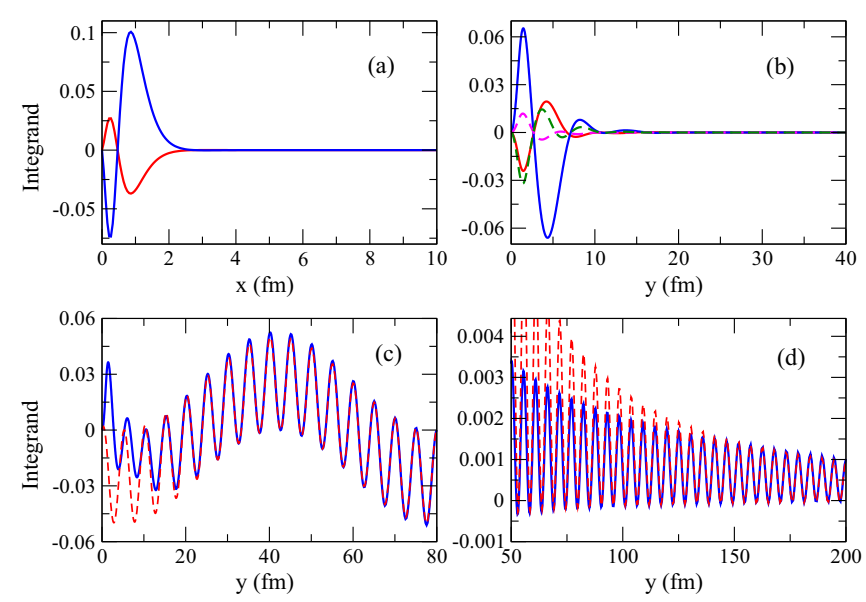

FIG. 2. (Color online) Typical integrand of Eq. (51) obtained for the $n-d$ reaction and for the followingcases: (a) as a function of $x$ for two fixed values of $y$; (b) as a function of $y$ ( $x$ coordinate integrated away), with $n$ being a $1+2$ channel for the neutron-neutron potential (solid curves) and for the neutron-proton potential with $s_{x}=0$ (dashed curves); (c) as a function of $y$ ( $x$ coordinate integrated away), with $n$ being a $1+2$ channel for the neutron-proton potential and $s_{x}=1$; and (d) as a function of $y$ ( $x$ coordinate integrated away), with $n$ being a breakup channel. In (c) and (d) dashed curves are the asymptotic matching given by Eqs. (54) and (59), respectively.

The integral over $y$ is, however, more complicated. In general, the integrand does not fall off exponentially at large distances, and its calculation is therefore more delicate. We can distinguish two different cases depending on the asymptotic behavior of the $f_{n 1}(\rho)$ radial wave function, i.e., when $n$ is associated with the $1+2$ channel or with a breakup channel. In the following we discuss the two cases.

\section{A. Index $\boldsymbol{n}$ corresponding to a $1+2$ channel}

When $n$ labels a $1+2$ channel ( $n=1$ in our case), the corresponding Faddeev amplitudes of the angular function $\Phi_{n}^{J M}\left(\rho, \Omega_{\rho}\right)$ behave at large distances as given by Eq. (E1), which reduces to

$$
\Phi_{n}^{J M}\left(\rho, \Omega_{i}\right) \rightarrow \rho^{3 / 2} \frac{1}{4 \pi} \Psi_{d}\left|\chi_{s_{d} s_{y}}^{J M}\right\rangle,
$$

when only $s$ waves are involved. In this expression $\Psi_{d}$ is the bound two-body wave function of the dimer associated with the $1+2$ channel $n$ and $s_{d}$ is the spin of the dimer.

Having this in mind, we can observe that the sum on the $\mathrm{HH}$ index $K$ in Eq. (51) can be reconstructed for $n=1(1+2$ channel) as

$$
\begin{aligned}
& \frac{1}{4 \pi} \sum_{K} C_{K, s_{x}}^{(n)}(\rho) N_{K} P_{\nu}^{\left(\frac{1}{2}, \frac{1}{2}\right)}(\cos 2 \alpha)\left\langle\chi_{s_{x} s_{y}}^{J M}|V(x)| \chi_{s_{x} s_{y}}^{J M}\right\rangle \\
& =\left\langle\Phi_{1}^{J M}\left(\rho, \Omega_{\rho}\right)|V(x)| \chi_{s_{x} s_{y}}^{J M}\right\rangle \\
& \quad=\sum_{i}\left\langle\Phi_{1}^{J M}\left(\rho, \Omega_{i}\right)|V(x)| \chi_{s_{x} s_{y}}^{J M}\right\rangle .
\end{aligned}
$$

The latter expression shows that, at large distances, the integrand in Eq. (51) contains explicitly the dimer wave function in the different Jacobi permutations. Two possibilities appear: the potential and the dimer depend on Jacobi coordinates belonging to two different permutations, for example, $\boldsymbol{x}_{i}$ and $\boldsymbol{x}_{j}$. In this case the integral in Eq. (51) has an exponential fall-off in both coordinates, $x$ and $y$. In fact, when the two-body potential refers to the two particles that do not form the dimer [for instance, the $V_{n n}(x)$ potential in the $n$ - $d$ case], large values of $y$ would correspond to large distances between the third particle and the one with which that third particle forms the dimer (large neutron-proton distance in the $n$ - $d$ case). Thus, for sufficiently large values of $y$ the integrand should be 0 due to the presence of the bound dimer wave function, which is now basically associated with the $y$ coordinates at large distances. Taking again the $n-d$ case as an illustration, the solid curves in Fig. 2(b) correspond to the integrand of Eq. (51) as a function of $y$ for two different values of the arclength $S$ (after integrating away the $x$ coordinate) for the case of the neutron-neutron potential. As shown in the figure, the integrand in the $y$ coordinate dies pretty quickly, and it is negligible already at distances of even less than $40 \mathrm{fm}$.

A similar case arises when the interaction is the one giving rise to the dimer (the neutron-proton potential in the $n$ - $d$ case) and $s_{x}$ is different from the spin of the bound dimer ( $s_{x}=0$ in the $n$ - $d$ case). The reason now is that the coefficients $C_{K, s_{x} \neq s_{d}}(\rho)$ in Eq. (51) go to 0 for large values of the hyperradius [as shown in Eq. (52), asymptotically only the terms with $s_{x}=s_{d}$ survive]. This case is illustrated in Fig. 2(b) by the dashed lines, which correspond to the $n$-d case and which show that also in this case the integrand goes to 0 rather quickly. Therefore, in the particular cases shown in Fig. 2(b) the numerical computation of the integral in both variables, $x$ and $y$, does not present particular problems.

A different situation appears when the potential in Eq. (51) and the dimer depend on the same variable $\boldsymbol{x}$ and when $s_{x}=s_{d}$ (neutron-proton potential and $s_{x}=1$ in the $n$ - $d$ case). In this case the the $y$-coordinate integrand in Eq. (51) does not vanish exponentially and a particular analysis of the integrand tail has to be performed.

It is well known [6] that the large-distance behavior of $f_{n 1}(\rho)$, for $n$ being a $1+2$ channel, is given by

$$
f_{n 1}(\rho) \rightarrow C \sin \left(k_{y}^{(n)} y\right)+D \cos \left(k_{y}^{(n)} y\right),
$$

where $C$ and $D$ are complex numbers (the $f_{n 1}$ functions are complex), and $\left(k_{y}^{(n)}\right)^{2}=2 m\left(E-E_{n}\right) / \hbar^{2}$, where $E_{n}$ is the binding energy of the dimer present in the $1+2$ channel associated with the adiabatic term $n$. Therefore, using Eqs. (54) and (52) it is clear that the integrand in Eq. (51) is asymptotically separable into $x$ and $y$ coordinates, and the integrand of the $y$ part goes like

Integrand $(y) \rightarrow C \sin \left(k_{y}^{(n)} y\right) \sin \left(k_{y} y\right)+D \cos \left(k_{y}^{(n)} y\right) \sin \left(k_{y} y\right)$,

where $C$ and $D$ are complex constants and where we have used that, since $x$ is restricted to small values, then $y / \rho \rightarrow 1$.

This behavior is shown in Fig. 2(c) again for the $n$ - $d$ case. The solid curve is the integrand in the $y$ coordinate obtained numerically for some value of the arclength $S$, and the dashed curve is the matching to the expression in Eq. (55). As shown 
in the figure the matching is pretty good already for distances of about 30-40 fm.

Therefore an easy way of computing the integral over $y$ in Eq. (51) (for the two-body potential giving rise to the dimer and $s_{x}=s_{d}$ ) is to do it numerically after subtracting the asymptotic behavior given in Eq. (55), i.e., integrating numerically the difference between the solid and the dashed curves in Fig. 2(c), which dies asymptotically sufficiently rapidly, and afterward add the analytical integral of Eq. (55) from $y=0$ to $y=\infty$. This analytical integral can be easily made by using that

$$
\begin{aligned}
& \int_{0}^{\infty} \sin \left(k_{y}^{(n)} y\right) \sin \left(k_{y} y\right) d y=\lim _{a \rightarrow 0}\left[\int_{0}^{\infty} e^{-a y} \sin \left(k_{y}^{(n)} y\right) \sin \left(k_{y} y\right) d y\right]=0 \quad(a>0) \\
& \int_{0}^{\infty} \cos \left(k_{y}^{(n)} y\right) \sin \left(k_{y} y\right) d y=\lim _{a \rightarrow 0}\left[\int_{0}^{\infty} e^{-a y} \cos \left(k_{y}^{(n)} y\right) \sin \left(k_{y} y\right) d y\right]=\frac{k_{y}}{\left(k_{y}\right)^{2}-\left(k_{y}^{(n)}\right)^{2}} \quad(a>0)
\end{aligned}
$$

where we have to remember that $k_{y}=\kappa \cos \alpha_{\kappa}$ and $k_{y}^{(n)}=$ $\sqrt{2 m\left(E-E_{n}\right) / \hbar^{2}}$, which are always different $\left(E_{n}<0\right)$.

\section{B. Index $\boldsymbol{n}$ corresponding to a breakup channel}

Due to the short-range character of the potential, the $x$ values are, in any case, restricted to relatively small values, no matter the character of the channel $n$ in Eq. (51). For this reason, even if $n$ corresponds to a breakup channel, the largedistance behavior of the integrand is given by contributions fulfilling that $y \gg x$ (or, in other words, $\rho \approx y$ ).

Also, for a breakup channel $n$, the coefficients $C_{K, s_{x}}^{(n)}(\rho)$ go to constant values [the angular eigenfunctions $\Phi_{n}^{J}$ become just a linear combination of HHs; see Eq. (28)], and the Jacobi polynomials $P_{\nu}^{\left(\frac{1}{2}, \frac{1}{2}\right)}(\cos 2 \alpha)$ contained in the HHs also go to the constant value $P_{\nu}^{\left(\frac{1}{2}, \frac{1}{2}\right)}(1)$. Finally, the general behavior at large distances of the radial functions $f_{n 1}(\rho)$ is given by a linear combination of the Hankel functions of first and second order [6], which means that their asymptotic behavior is given by

$$
f_{n 1}(\rho) \rightarrow \sum_{m=0}^{M} \frac{C_{m} \sin (\kappa y)+D_{m} \cos (\kappa y)}{y^{m}},
$$

where we have already replaced $\rho$ with $y$.

Therefore, when $n$ is associated with a breakup channel, the $y$ part of the integrand in Eq. (51) goes asymptotically as

$$
\begin{aligned}
& \text { Integrand }(y) \\
& \qquad \sum_{m=0}^{M}\left(\frac{C_{m} \sin (\kappa y) \sin \left(k_{y} y\right)}{y^{m+3 / 2}}+\frac{D_{m} \cos (\kappa y) \sin \left(k_{y} y\right)}{y^{m+3 / 2}}\right),
\end{aligned}
$$

where, again, the constants $C_{m}$ and $D_{m}$ are complex and $k_{y}=$ $\kappa \cos \alpha_{\kappa}$. Obviously, the higher the value of $M$, the lower the value of $y$ at which the matching with the numerical integrand is obtained.

Thus, for outgoing breakup channels, the $y$ part of the integral in Eq. (51) goes to 0 asymptotically as $1 / y^{3 / 2}$. It could then seem that such an integral could be obtained numerically without much trouble. However, the fall-off is not fast enough, and at relatively large distances the integrand is not really negligible. Again, taking the $n-d$ case as an example, this is shown in Fig. 2(d), where the solid (blue) curve is the integrand in Eq. (51) as a function of $y$ for some value of the arclength $S$ and with $n$ being one of the breakup channels $(n>1)$. At a distance of about $200 \mathrm{fm}$ the integrand is not at all negligible; in fact the amplitude of the oscillations shown in the figure are still about $10 \%$ of the maximum computed amplitude. The dashed (red) curve shows the matching given by Eq. (59) and $M=0$. It is clear that this matching is less accurate than the one shown in Fig. 2(c), and the agreement now with the true integrand is observed much farther, at $150 \mathrm{fm}$ at least.

Therefore, due to the too slow fall-off of the integrand, it is convenient to integrate numerically up to some $y_{\max }$ value at which the matching with the asymptotic behavior in Eq. (59) has already been achieved and to perform analytically the integrand from $y_{\max }$ up to $\infty$. These analytical integrations involve the so-called Fresnel integrals, and they can be performed as indicated in Appendix F.

\section{THE $n-d$ CASE}

In this section we compute the cross sections for $n$-d breakup. This choice is made to enable comparison with the benchmark calculations given in [18]. Following this reference, we have chosen the Malfliet-Tjon-I-III model $s$ wave nucleon-nucleon potential [20], which, for the triplet and singlet cases, takes the form

$$
V_{t}(r)=\frac{1}{r}\left(-626.885 e^{-1.55 r}+1438.72 e^{-3.11 r}\right)
$$

and

$$
V_{s}(r)=\frac{1}{r}\left(-513.968 e^{-1.55 r}+1438.72 e^{-3.11 r}\right),
$$

respectively, and where the $r$ is in fm, the potential in $\mathrm{MeV}$, and $\hbar^{2} / m=41.47 \mathrm{MeV} \mathrm{fm}^{2}$. In our calculations $m$ is taken as the normalization mass in the definitions of the Jacobi coordinates given in Eqs. (3) to (6).

In Sec. IV A we summarize the results obtained in Ref. [6] for the $\mathcal{S}$ matrix after application of the integral relations. We continue in Sec. IV B with the cross sections obtained from the adiabatic expansion of the transition amplitude in Eq. (30), and the inaccuracies corresponding to some particular geometries are analyzed. In Sec. IV C we show how the use of the transition amplitude given by Eq. (46) corrects the cross sections in the regions where inaccuracies were observed. 
TABLE I. Inelasticity parameter $\left|\mathcal{S}_{11}\right|$ for neutron-deuteron scattering for two laboratory neutron beam energies (14.1 and $42.0 \mathrm{MeV})$ for the doublet and quartet cases. The value of $K_{\max }$ is the $K$ value associated with the last adiabatic potential included in the calculation. The last row gives the value quoted in Ref. [18].

\begin{tabular}{lccccc}
\hline \hline \multirow{2}{*}{$K_{\max }$} & \multicolumn{2}{c}{ Doublet } & & \multicolumn{2}{c}{ Quartet } \\
\cline { 2 - 3 } \cline { 5 - 6 } & $14.1 \mathrm{MeV}$ & $42.0 \mathrm{MeV}$ & & $14.1 \mathrm{MeV}$ & $42.0 \mathrm{MeV}$ \\
\hline 4 & 0.4662 & 0.4929 & & 0.9794 & 0.8975 \\
8 & 0.4637 & 0.4993 & & 0.9784 & 0.9026 \\
12 & 0.4640 & 0.5014 & & 0.9783 & 0.9030 \\
16 & 0.4643 & 0.5019 & & 0.9782 & 0.9031 \\
20 & 0.4644 & 0.5021 & & 0.9782 & 0.9033 \\
24 & 0.4645 & 0.5022 & & 0.9782 & 0.9033 \\
28 & 0.4645 & 0.5022 & & 0.9782 & 0.9033 \\
Ref. [18] & 0.4649 & 0.5022 & & 0.9782 & 0.9033 \\
\hline \hline
\end{tabular}

\section{A. $\mathcal{S}$ matrix}

The details concerning the integral relations formalism applied to the description of breakup $1+2$ reactions are given in Ref. [6]. This method permits us to extract the $\mathcal{S}$ matrix of the reaction from the internal part of the wave function. When this wave function is obtained by means of the adiabatic expansion method, the pattern of convergence of the $\mathcal{S}$ matrix is similar to the one obtained with the same method for bound states.

In [6] the integral relations method is applied to the $n$ $d$ reaction. Only $s$ waves are considered in the calculation, which implies that only two different total angular momenta are possible: the quartet case $(J=3 / 2)$, for which only the triplet $s$-wave potential in Eq. (60) enters; and the doublet case $(J=1 / 2)$, for which both the singlet and the triplet potentials contribute.

The unitarity of the $\mathcal{S}$ matrix implies that, given an incoming channel, for instance, channel 1 ( $n$ - $d$ channel), we have that $\sum_{n=1}^{\infty}\left|\mathcal{S}_{1 n}\right|^{2}=1$ or, in other words,

$$
\sum_{n=2}^{\infty}\left|\mathcal{S}_{1 n}\right|^{2}=1-\left|\mathcal{S}_{11}\right|^{2},
$$

which means that an accurate calculation of the elastic term $\mathcal{S}_{11}$ amounts to an accurate calculation of the infinite summation of the $\left|\mathcal{S}_{1 n}\right|^{2}$ terms $(n>1)$ corresponding to the breakup channels and, therefore, allowing the calculation of the total breakup cross section given in Eq. (2).

The complex value of $\mathcal{S}_{11}$ can be written in terms of a complex phase shift $\delta$ as

$$
\mathcal{S}_{11}=e^{2 i \delta}=e^{-2 \operatorname{Im}(\delta)} e^{2 i \operatorname{Re}(\delta)}=\left|\mathcal{S}_{11}\right| e^{2 i \operatorname{Re}(\delta)} .
$$

The value of $\left|\mathcal{S}_{11}\right|^{2}$ gives the probability of elastic $n$-d scattering, and $\left|\mathcal{S}_{11}\right|$ is what is usually referred to as the inelasticity parameter (denoted $\eta$ in [18] and [20]). Obviously, the closer the inelasticity is to 1 , the more elastic the reaction. In fact, for energies below the breakup threshold the phase shift is real and $\left|\mathcal{S}_{11}\right|=1$.

In Tables I and II we list the results given in [6] for the inelasticity parameter $\left|\mathcal{S}_{11}\right|$ and the real part of the phase shift
TABLE II. The same as Table I, for $\operatorname{Re}(\delta)$.

\begin{tabular}{lccccc}
\hline \hline \multirow{2}{*}{$K_{\max }$} & \multicolumn{2}{c}{ Doublet } & & \multicolumn{2}{c}{ Quartet } \\
\cline { 2 - 3 } \cline { 5 - 6 } & $14.1 \mathrm{MeV}$ & $42.0 \mathrm{MeV}$ & & $14.1 \mathrm{MeV}$ & $42.0 \mathrm{MeV}$ \\
\hline 4 & 105.82 & 42.66 & & 69.04 & 38.98 \\
8 & 105.57 & 41.65 & & 68.99 & 37.95 \\
12 & 105.53 & 41.49 & & 68.98 & 37.77 \\
16 & 105.53 & 41.46 & & 68.97 & 37.73 \\
20 & 105.53 & 41.45 & & 68.96 & 37.72 \\
24 & 105.53 & 41.44 & & 68.96 & 37.71 \\
28 & 105.53 & 41.44 & & 68.96 & 37.71 \\
Ref. [18] & 105.50 & 41.37 & & 68.96 & 37.71 \\
\hline \hline
\end{tabular}

$\operatorname{Re}(\delta)$, respectively. We have used the same two laboratory energies considered in Ref. [18], i.e., 14.1 and 42.0 MeV. The value of $K_{\max }$ given in the tables corresponds to the asymptotic grand-angular quantum number associated with the last adiabatic potential included in the adiabatic expansion.

As reported in the tables, the agreement with the results in Ref. [18] is good. Actually, we obtain precisely the same result for the two incident energies in the quartet case and a small difference, clearly smaller than $0.1 \%$, in the doublet case. Furthermore, the pattern of convergence is rather rapid, especially in the quartet case, for which already for $K_{\max }=8$ we obtain a result that can be considered very accurate. In the doublet case the convergence is a bit slower, and a value of $K_{\text {max }}$ of about 16 is needed.

\section{B. Cross sections}

Once the $\mathcal{S}$ matrix of the reaction has been computed, we can obtain the transition matrix according to the expression in Eq. (30) and, therefore, also the expression, Eq. (31), and the lab cross section in Eq. (14).

We shall consider an incident energy in the laboratory frame of $E_{i n}^{(\mathrm{lab})}=14.1 \mathrm{MeV}$ and four different outgoing geometries specified by the polar angles $\theta_{1}$ and $\theta_{2}$ and the difference of azimuthal angles $\Delta \varphi=\varphi_{1}-\varphi_{2}$, where $\left(\theta_{1}, \varphi_{1}\right)$ and $\left(\theta_{2}, \varphi_{2}\right)$ describe the direction of the two outgoing neutrons. The four different cases are as follows.

Case 1: $\theta_{1}=45.0^{\circ}, \theta_{2}=53.56^{\circ}, \Delta \varphi=180^{\circ}$.

Case 2: $\theta_{1}=35.0^{\circ}, \theta_{2}=44.0^{\circ}, \Delta \varphi=180^{\circ}$.

Case 3: $\theta_{1}=60.06^{\circ}, \theta_{2}=53.10^{\circ}, \Delta \varphi=180^{\circ}$.

Case 4: $\theta_{1}=51.02^{\circ}, \theta_{2}=51.02^{\circ}, \Delta \varphi=120^{\circ}$.

The solid lines in Fig. 3 show the computed cross section in Eq. (14) for the four cases given above for the quartet case $(J=3 / 2)$. In the figure, $K_{\max }$ refers to the asymptotic value of the grand-angular quantum number $K$ associated with the last adiabatic term included in the expansion in Eq. (30). A value of $K_{\max }=34$ amounts to including 18 adiabatic terms in the expansion. This is enough to reach convergence. In fact, the same calculation with $K_{\max }=22$ (12 adiabatic terms) is basically indistinguishable from the curves shown in the figure. The cross sections given in the benchmark calculation in Ref. [18] are shown by the filled-circle curves. As we can 


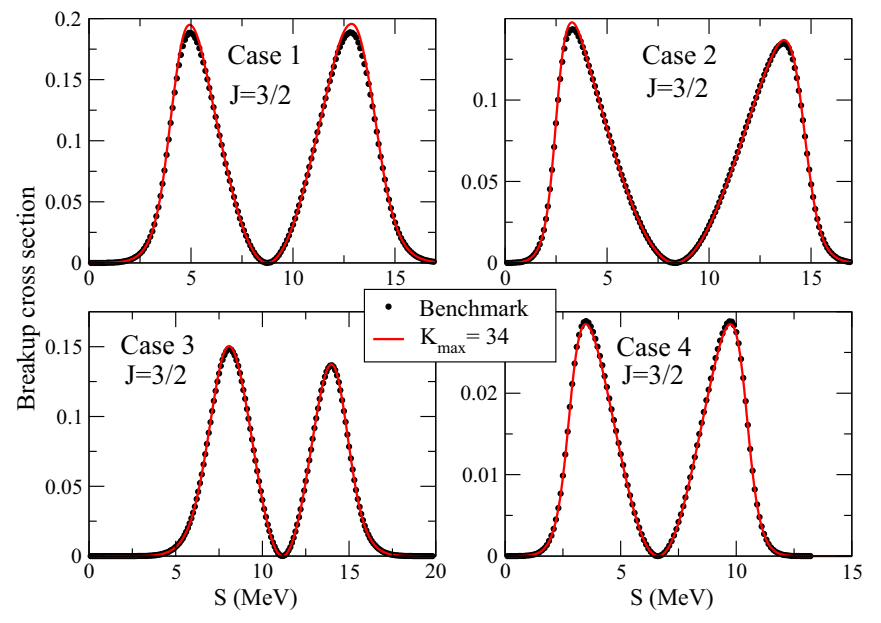

FIG. 3. (Color online) Breakup cross sections, Eq. (14), as a function of the arclength $S$ for $n$ - $d$ scattering in the quartet case $(J=3 / 2)$ for a laboratory incident energy of $14.1 \mathrm{MeV}$. Cross sections are given in $\mathrm{mb} /\left(\mathrm{MeV} \mathrm{sr}{ }^{2}\right)$. The value of $K_{\max }$ refers to the grand-angular quantum number $K$ associated with the last adiabatic term included in the expansion in Eq. (30). The four cases shown correspond to the four directions of the outgoing neutrons specified in the text. The filled-circle curves are the result of the benchmark calculations given in Ref. [18].

see, the results given in the benchmark calculation are nicely reproduced.

The corresponding cross sections for the doublet case ( $J=1 / 2)$ are shown by the dashed curves in the upper panels in Figs. 4 and 5. When the contribution from the quartet state (Fig. 3) is added, we get the total cross section given by the solid curves in the same figures. The doublet cross sections have been obtained with $K_{\max }=34$, which, for $J=1 / 2$,
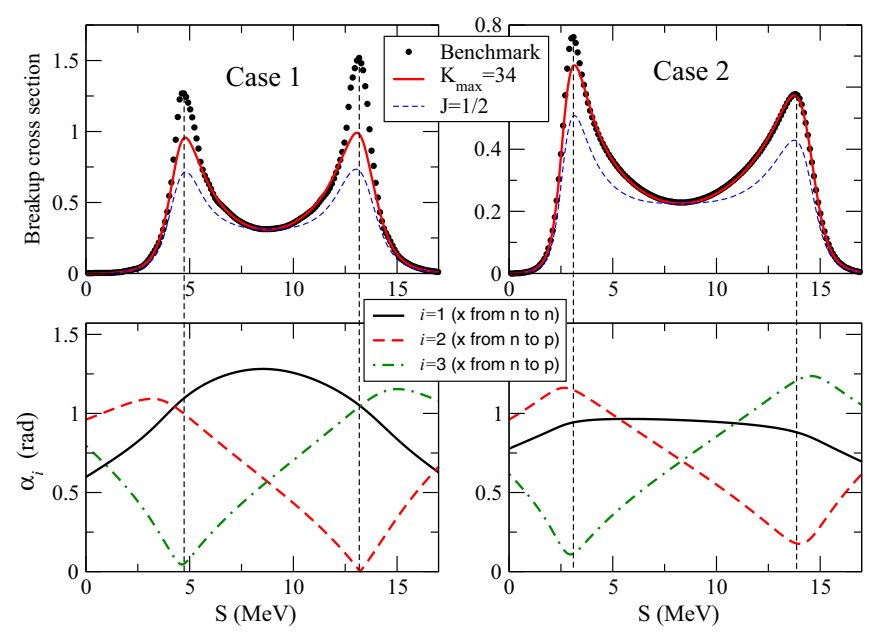

FIG. 4. (Color online) Top: Total cross sections for cases 1 and 2 in the upper panels in Fig. 3. They are obtained by adding the quartet $(J=3 / 2)$ contribution in Fig. 3 and the doublet $(J=1 / 2)$ contribution shown by the dashed curves. Bottom: For the same two cases, the corresponding values of $\alpha_{i}(i=1,2,3)$ as a function of the arclength $S$.
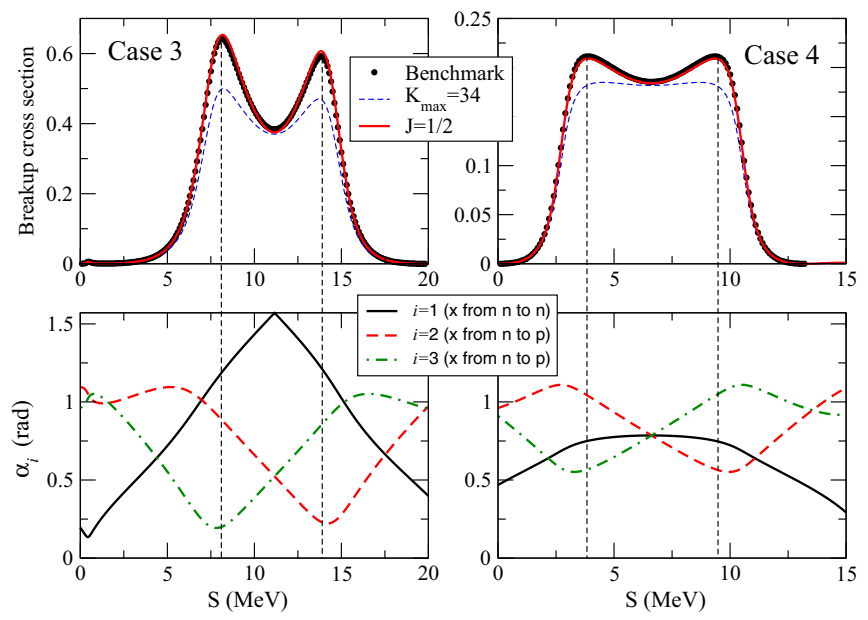

FIG. 5. (Color online) The same as Fig. 4, for cases 3 and 4.

corresponds to inclusion of 35 adiabatic terms in the expansion of Eq. (30). Again, when $K_{\max }$ is reduced to 22 (23 adiabatic terms) the computed curves cannot be distinguished from the ones shown in the figure. As we can see, even if the computed cross sections have converged, there is a clear discrepancy from the benchmark calculation for case 1 and, to a lower extent, for case 2 (Fig. 4). For cases 3 and 4 (Fig. 5) the agreement is reasonably good.

As anticipated in Sec. IID, the discrepancy from the benchmark calculation appears in the $S$ regions corresponding to an outgoing kinematics where two of the particles have zero relative energy. This is shown in Fig. 4, where the lower panels show, as a function of the arclength $S$, the hyperangle $\alpha_{i}=\arctan \left(k_{x_{i}} / k_{y_{i}}\right)$ for cases 1 and 2 . The index $i=1$ corresponds to the Jacobi coordinates where $\boldsymbol{x}$ connects the two neutrons (solid curve), and the indices $i=2,3$ correspond to the Jacobi coordinates where $\boldsymbol{x}$ connects the proton and one of the neutrons (dashed and dot-dashed curves). For case 1 (left panels), we can see that $\alpha_{2}$ and $\alpha_{3}$ approach 0 very often for $S$ slightly below $5 \mathrm{MeV}$ and about $13 \mathrm{MeV}$. In these regions the proton and one of the neutrons move with relative zero (or very small) energy, and these are precisely the regions where the discrepancy from the benchmark calculation is observed. For case 2, $\alpha_{2}$ and $\alpha_{3}$ do not approach 0 as often as in case 1 , and the discrepancy from the benchmark calculation is now much smaller than in case 1 (or even not visible, as happens for the second peak in the cross section).

For completeness, we show in the lower panels in Fig. 5 the same data as in Fig. 4, but for cases 3 and 4. Now the values of $\alpha_{i}$ do not reach 0 for any $S$ value, and the agreement between our calculation and the benchmark is fine for all values of $S$.

As mentioned, the problem is related to the fact that $\alpha_{i} \approx 0$ means two of the particles flying together with relative zero (or very low) energy and the third particle moving far apart. The adiabatic expansion in Eq. (30) is then trying to reproduce the zero-energy two-body wave function (which is proportional to $\sin \alpha$ ) in terms of a set of polynomials (the Jacobi polynomials) that have $\cos 2 \alpha$ as the argument. A correct description of the zero-energy two-body wave function would require, then, infinitely many adiabatic terms. 
As shown in Fig. 3, the inaccuracy mentioned above for $\alpha_{i} \approx 0$ is not visible in the quartet case, where only triplet nucleon-nucleon components enter. However, as shown in Fig. 4, where the contribution from the doublet has been added, the mismatch with the benchmark calculation becomes very significant. This is due to the fact that in the doublet case there is an important contribution from the singlet nucleon-nucleon components. This small difference is actually very relevant, because the singlet nucleon-nucleon potential has the rather large scattering length of about $-23 \mathrm{fm}$, more than four times larger (in absolute value) than the scattering length of the triplet nucleon-nucleon potential (about $5 \mathrm{fm}$ ). This means that the $s$-wave singlet nucleon-nucleon system has a pretty low-lying virtual state that favors the structure mentioned above of two nucleons flying together after the collision at a very low relative energy. For the case of the triplet nucleon-nucleon state the energy of the corresponding virtual state is about 20 times higher than in the singlet case (the virtual-state energy is proportional to the inverse of the scattering length squared).

The solution suggested in order to solve the disagreement shown in Fig. 4 is to skip the expansion of the outgoing wave in terms of the different adiabatic channels and compute instead the transition amplitude as given in Eq. (46), where the adiabatic expansion does not enter. The results obtained using this alternative method are given in the following section.

\section{Corrections to the cross sections}

The cross section in Eq. (15) is now computed making use of Eq. (46). The matrix element in this expression is given by Eq. (51), whose calculation requires use of the techniques described in Secs. III A and III B when integrating over the $y$ coordinate. For the case illustrated in Fig. 2(c) the integral over $y$ is made by integrating numerically the difference between the computed integrand and its analytic asymptotic behavior [difference between solid and dashed curves in Fig. 2(c)] and adding the analytical integral, from 0 to $\infty$, of the asymptotic behavior. These analytical integrals can be obtained with the help of Eqs. (56) and (57). For the case described in Fig. 2(d) the integral over $y$ is made by integrating numerically up to some $y_{\max }$ at which the asymptotic behavior in Eq. (59) has already been reached, and the integral from $y_{\max }$ to $\infty$ is made as described in Appendix F.

The integral in Eq. (51) contains a summation over the adiabatic terms $n$ and the grand-angular quantum number $K$. This double-summation comes from the expansion of the incident three-body wave function $\Psi_{1}$ given in Eq. (48). The convergence in terms of $n$ has been found to be fast, and use of about 20 adiabatic terms is enough to get a converged result. However, the summation over $K$ is much more delicate. In fact, in the $S$ regions where the discrepancy between the old calculation and the benchmark was found, the number of $\mathrm{HHs}$ needed in Eq. (51) can be pretty high.

This is shown in Fig. 6, where we show the same cross sections as in Fig. 4 for cases 1 and 2. The benchmark result is given by the filled circles and the old computed cross sections, as shown in Fig. 4, are given by the dashed curves. The cross sections obtained with the new procedure have been computed including 35 adiabatic terms in the expansion in Eq. (51), and
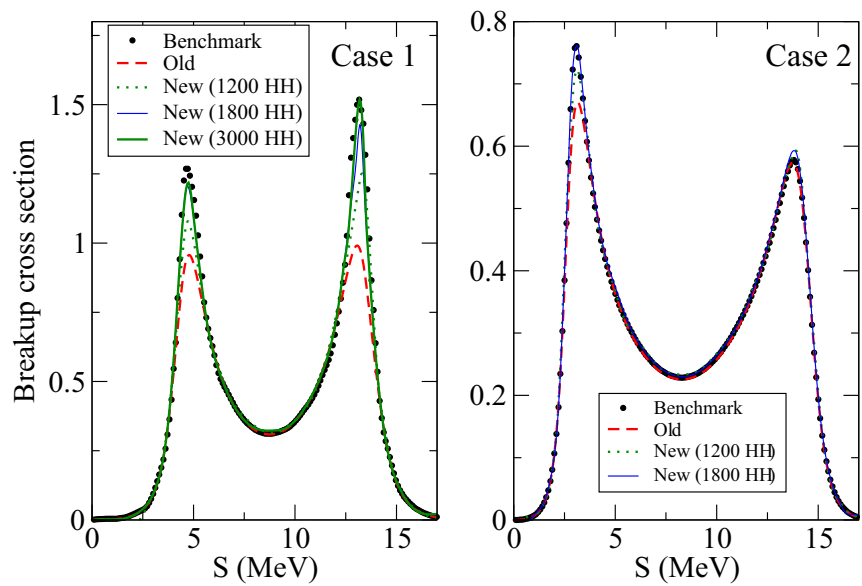

FIG. 6. (Color online) Total cross sections [in $\left.\mathrm{mb} /\left(\mathrm{MeV} \mathrm{sr}^{2}\right)\right]$ for cases 1 and 2 shown in the upper panels in Fig. 4 [ $\left.E_{\text {in }}^{(\text {lab })}=14.1 \mathrm{MeV}\right]$. Filled circles are the benchmark calculations from Ref. [18]. Dashed curves are the results shown in Fig. 4, which were computed using the expansion in Eq. (30). Cross sections obtained by means of Eq. (46) are shown by the filled-circle, thin solid, and thick solid curves, which correspond, respectively, to calculations including 1200, 1800, and 3000 hyperspherical harmonics when computing the integral in Eq. (51).

the numbers of HHs used in the same expansion are 1200 for the dotted curves, 1800 for the thin solid curves, and 3000 for the thick solid curve.

Needless to say, when the same procedure is used to compute the cross sections forcases 3 and 4, the same results as thoseshown for these two cases in Fig. 5 are obtained. As we can see, in the regions where the results shown in Fig. 3 matched the benchmark, the new calculations still reproduce the results equally well. However, in the peaks where the old calculation and the benchmark did not agree, the new calculation improves the agreement significantly. As already mentioned, agreement is actually reached when a sufficiently high number of HHs is included in the calculation. In fact, as we can see, $1200 \mathrm{HHs}$ are still not enough, and at least 1800 of them are needed in case 2, and 3000 in case 1.

For completeness, we also show the results for the two benchmark calculations given in Ref. [18] corresponding to an incident energy in the laboratory frame of $42.0 \mathrm{MeV}$. The angles describing the direction of the outgoing neutrons in these two cases are as follows.

Case 5: $\theta_{1}=45.0^{\circ}, \theta_{2}=60.54^{\circ}, \Delta \varphi=180^{\circ}$.

Case 6: $\theta_{1}=53.61^{\circ}, \theta_{2}=53.61^{\circ}, \Delta \varphi=120^{\circ}$.

The results for cases 5 and $6\left[E_{\text {in }}^{(\text {lab })}=42.0 \mathrm{MeV}\right]$ are shown in Fig. 7. As in Figs. 4 and 5, the lower panels show the values of the hyperangle $\alpha_{i}$ as a function of the arclength $S$. As we can see, case 5 given above is analogous to cases 1 and 2 shown in Fig. 4, where the hyperangles $\alpha_{2}$ and $\alpha_{3}$ approach 0 for certain values of the arclength $S$. Now this happens for values of $S$ about 13 and $40.5 \mathrm{MeV}$. For this reason, an accurate calculation of the cross section for case 5 in the vicinity of these two $S$ values requires use of the expansion given in Eq. (46). For case 6 the value of the hyperangle $\alpha$ is always far from 0 (as in 


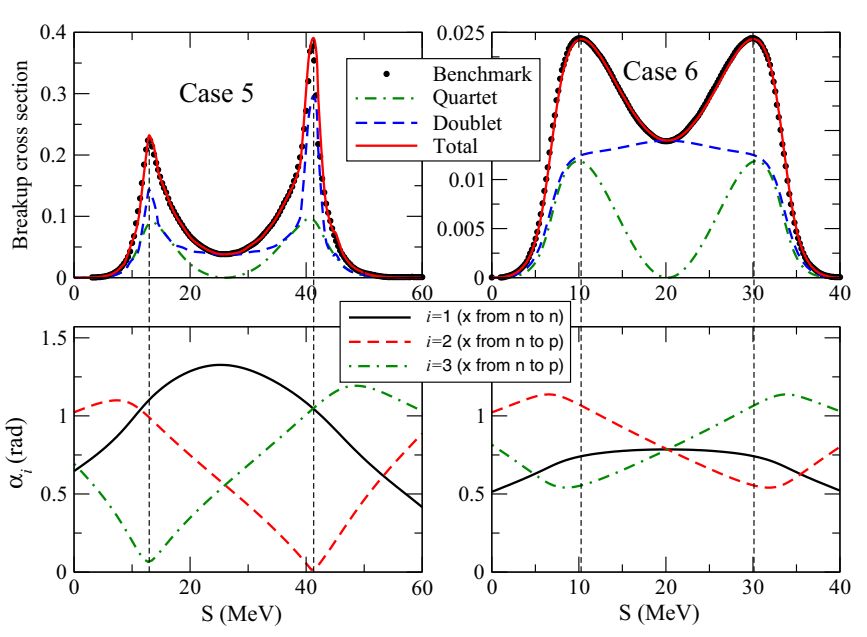

FIG. 7. (Color online) Top: Total cross sections [in mb/(MeV $\left.\mathrm{sr}^{2}\right)$ ] for cases 5 and 6 and $E_{\mathrm{in}}^{(\mathrm{lab})}=42.0 \mathrm{MeV}$ (see text). Filled circles are the benchmark calculations from Ref. [18]. Dot-dashed and dashed curves are the quartet $(J=3 / 2)$ and doublet $(J=1 / 2)$ contributions, respectively. Total cross sections are given by solid curves. For case 6 (right panel) the calculation using the expansion in Eq. (30) is accurate enough. For case 5 (left panel) the expansion in Eq. (46) is required in order to obtain sufficient accuracy in the vicinity of the peaks. Bottom: For the same two cases, the corresponding values of $\alpha_{i}(i=1,2,3)$ as a function of the arclength $S$.

cases 3 and 4 shown in Fig. 5), and use of the expansion in Eq. (30) is enough to get a sufficient accuracy in the calculations.

The panels in Fig. 7 shows the corresponding cross sections. The dot-dashed and dashed curves are the quartet $(J=3 / 2)$ and doublet $(J=1 / 2)$ contributions, respectively. The total cross section is given by the solid curves. As we can see, the agreement with the benchmark calculations (filled circles) given in Ref. [18] is very good. In case 5 the convergence in the vicinity of the two peaks, computed through Eq. (46), has required the inclusion of up to 40 adiabatic terms in the expansion of the three-body wave function [see Eq. (20)]. This number is higher than in the calculations for $E_{\text {in }}^{(\mathrm{lab})}=14.1 \mathrm{MeV}$, where even fewer than 30 adiabatic channels was enough. This is because for a given value of the hyperradius $\rho$, only adiabatic potentials whose value at $\rho$ is lower than the incident energy (or close enough to the energy, if they are above) contribute to the radial wave functions $f_{n 1}(\rho)$ obtained through Eq. (21). Obviously, the higher the three-body energy $E$, the higher the number of adiabatic potentials contributing to the radial wave functions.

\section{SUMMARY AND CONCLUSIONS}

The description of three-body systems, in particular, of $1+2$ reactions, by use of the adiabatic expansion method has been proved to be efficient provided that the integral relations introduced in Ref. [3] are used. Because of these two relations the $\mathcal{S}$ matrix of the reaction can be extracted from the internal part of the wave function, and subsequently the pattern of convergence of the adiabatic expansion is fast. Knowledge of the $\mathcal{S}$ matrix then permits usn to obtain accurate results for the total cross sections for the different open channels (elastic, inelastic, transfer, and breakup).

However, even if the $\mathcal{S}$ matrix and the total cross sections can be accurately computed, it is not obvious that the differential cross sections that one extracts from the $\mathcal{S}$ matrix are also accurate. In fact, calculation of the differential cross sections requires knowledge not only of the $\mathcal{S}$ matrix, but of the full transition amplitude. When the adiabatic expansion is applied to obtain the transition amplitude, the number of adiabatic terms necessary in order to achieve convergence strongly depends on the geometry of the outgoing particles, and in some circumstances this number can be exceedingly large.

In this work we have given the details of how to compute differential cross sections for $1+2$ reactions above the threshold for breakup of the dimer. The expressions that permit us to transform the cross sections from the center-of-mass frame to the laboratory frame have been derived. The three-body wave function describing the three-body system, as well as the transition amplitude, is computed by use of the adiabatic expansion method.

The case of $n$ - $d$ breakup has been taken as a test, and our results have been compared with the benchmark calculations described in Refs. [18] and [20]. Six different geometries have been considered for the three outgoing nucleons after the breakup.

In general, the agreement with the benchmark results has been found to be good. The only discrepancy appears in those cases corresponding to two outgoing particles flying away together after the breakup with zero relative energy. In this case the adiabatic expansion of the transition amplitude is not convenient, since the number of adiabatic terms needed to reproduce this structure is too large.

We have shown that this problem can be solved by skipping the partial-wave expansion of the outgoing wave and expressing the transition amplitude in terms of a full, nonexpanded, outgoing plane wave. This calculation is much more demanding from the numerical point of view, and special care has to be taken with the number of HHs used to describe the incoming wave function.

This study can be seen as a first step in the inclusion of the Coulomb interaction in the study of $1+2$ nuclear processes. Preliminary studies using the HA expansion have been done in Ref. [5] for the elastic channel of the proton-deuteron reaction. In the breakup case, Eq. (51) cannot be applied directly since the free-plane wave was used in its derivation, and it should be replaced by a distorted wave due to the long range of the Coulomb potential. This study is under way at present, following the analysis given in Ref. [17]. However, it should be noted that the configuration in which two protons move away together is suppressed and the one in which a neutron-proton pair moves away together far from the second proton shows tiny Coulomb effects (see Ref. [21]). Hence, we expect to extend the analysis presented here to the proton-deuteron case and, in general, to $1+2$ nuclear processes, allowing us to analyze several reactions of astrophysical interest in which the inclusion of the Coulomb interaction cannot be avoided. 


\section{ACKNOWLEDGMENT}

This work was partly supported by funds provided by DGI of MINECO (Spain) under Contract No. FIS2011-23565.

\section{APPENDIX A: THE OUTGOING FLUX}

The time-dependent Schrödinger equation in hyperspherical coordinates is given by [1]

$$
i \hbar \frac{\partial \Psi}{\partial t}=\frac{\hbar^{2}}{2 m}\left(-\frac{\partial^{2} \Psi}{\partial \rho^{2}}-\frac{5}{\rho} \frac{\partial \Psi}{\partial \rho}\right)+\frac{\hbar^{2}}{2 m} \frac{\hat{\Lambda}}{\rho^{2}} \Psi+\hat{V} \Psi,
$$

where $\Psi$ is the three-body wave function, $\hat{\Lambda}$ is the grandangular operator whose eigenfunctions are the HHs, and $\hat{V}$ is the operator containing the two-body potentials. The selfadjoint of the equation above is just

$$
-i \hbar \frac{\partial \Psi^{\dagger}}{\partial t}=\frac{\hbar^{2}}{2 m}\left(-\frac{\partial^{2} \Psi^{\dagger}}{\partial \rho^{2}}-\frac{5}{\rho} \frac{\partial \Psi^{\dagger}}{\partial \rho}\right)+\frac{\hbar^{2}}{2 m} \Psi^{\dagger} \frac{\hat{\Lambda}}{\rho^{2}}+\Psi^{\dagger} \hat{V},
$$

Multiplying Eq. (A1) by $\Psi^{\dagger}$ from the left and Eq. (A2) by $\Psi$ from the right we get two expressions that, after subtracting, lead to

$$
\rho^{5} \frac{\partial|\Psi|^{2}}{\partial t}=-\frac{1}{i} \frac{\hbar}{2 m} \frac{\partial}{\partial \rho}\left[\Phi^{\dagger} \frac{\partial \Phi}{\partial \rho}-\frac{\partial \Phi^{\dagger}}{\partial \rho} \Phi\right],
$$

where $\Phi=\rho^{5 / 2} \Psi$. Therefore, the number of particles per unit time through the unit of surface of a hypersphere of hyperradius $\rho$ is given by

$$
\frac{1}{i} \frac{\hbar}{2 m} \frac{1}{\rho^{5}}\left[\Phi^{\dagger} \frac{\partial \Phi}{\partial \rho}-\frac{\partial \Phi^{\dagger}}{\partial \rho} \Phi\right]
$$

Thus, the flux of particles through an element of hypersurface $d \Sigma$ is given by

$$
\text { outgoing flux }=\frac{1}{i} \frac{\hbar}{2 m} \frac{1}{\rho^{5}}\left[\Phi^{\dagger} \frac{\partial \Phi}{\partial \rho}-\frac{\partial \Phi^{\dagger}}{\partial \rho} \Phi\right] d \Sigma .
$$

The asymptotic behavior of the three-body wave function in hyperspherical coordinates takes the form

$$
\Psi \stackrel{\rho \rightarrow \infty}{\longrightarrow} \frac{1}{\rho^{5 / 2}} e^{i \kappa \rho} A
$$

where $\kappa=\sqrt{2 m E} / \hbar, E$ is the total three-body energy, and $A$ is the transition amplitude. Keeping in mind that $\Phi=\rho^{5 / 2} \Psi$, substituting Eq. (A6) into Eq. (A5), and using that the element of hypersurface is given by Eq. (8), we get the following final expression for the outgoing flux:

$$
\text { outgoing flux }=\hbar \frac{\kappa}{m}|A|^{2}\left(\frac{m}{\mu_{x}}\right)^{3 / 2}\left(\frac{m}{\mu_{y}}\right)^{3 / 2} d \Omega_{\kappa},
$$

where we have used that, asymptotically, $d \Omega=d \Omega_{\kappa}$.

\section{APPENDIX B: PHASE SPACE FOR THREE PARTICLES WITH EQUAL MASS}

The choice of three particles with equal mass is made just to simplify the notation and the expressions derived in the following subsections. The mass of the particles $m$ is also taken as the normalization mass in the definition of the Jacobi coordinates given in Eqs. (3) to (6). In any case the extension of the expressions below to particles with different masses is straightforward.

\section{Center-of-mass frame}

To write down the phase space in the center-of-mass frame we use the momentum coordinates $\left\{\boldsymbol{p}_{x}, \boldsymbol{p}_{y}, \boldsymbol{P}\right\}$, where $\boldsymbol{p}_{x}$ and $\boldsymbol{p}_{y}$ are defined in Eqs. (5) and (6), and $\boldsymbol{P}=\boldsymbol{p}_{i}+\boldsymbol{p}_{j}+\boldsymbol{p}_{k}$ is the momentum of the three-body center of mass. In these coordinates the phase space takes the form

$$
\begin{aligned}
\Phi= & \int d \boldsymbol{p}_{x} d \boldsymbol{p}_{y} d \boldsymbol{P} \delta\left(E-\frac{\hbar^{2} p_{x}^{2}}{2 \mu_{x}}-\frac{\hbar^{2} p_{y}^{2}}{2 \mu_{y}}\right) \delta^{(3)} \\
& \times\left(\boldsymbol{P}-\boldsymbol{p}_{i}-\boldsymbol{p}_{j}-\boldsymbol{p}_{k}\right)
\end{aligned}
$$

where the $\delta$ functions impose energy and momentum conservation ( $E$ is the three-body energy in the center-of-mass frame).

The integration in (B1) over $\boldsymbol{P}$ can be made trivially thanks to the momentum $\delta$ function, and the phase space can be rewritten as

$$
\Phi=\left(\frac{\mu_{x}}{m}\right)^{3 / 2}\left(\frac{\mu_{y}}{m}\right)^{3 / 2} \int \kappa^{5} d \kappa d \Omega_{\kappa} \delta\left(E-\frac{\hbar^{2} \kappa^{2}}{2 m}\right),
$$

where we have used that, according to Eqs. (5) and (6),

$$
d \boldsymbol{p}_{x} d \boldsymbol{p}_{y}=\left(\frac{\mu_{x}}{m}\right)^{3 / 2}\left(\frac{\mu_{y}}{m}\right)^{3 / 2} d \boldsymbol{k}_{x} d \boldsymbol{k}_{y}
$$

and that $d \boldsymbol{k}_{x} d \boldsymbol{k}_{y}=\kappa^{5} d \kappa d \Omega_{\kappa}$, where $\Omega_{\kappa}$ represents the hyperangles in momentum space.

The $\delta$ function in Eq. (B2) can be rewritten as

$$
\delta\left(E-\hbar^{2} \frac{\kappa^{2}}{2 m}\right)=\frac{m}{\hbar^{2} \kappa} \delta\left(\kappa-\frac{\sqrt{2 m E}}{\hbar}\right),
$$

which means that, after integration over $\kappa$, the phase space, (B2), takes the final form

$$
d^{5} \Phi=\left(\frac{\mu_{x}}{m}\right)^{3 / 2}\left(\frac{\mu_{y}}{m}\right)^{3 / 2} \kappa^{4} \frac{m}{\hbar^{2}} d \Omega_{\kappa} .
$$

\section{Laboratory frame}

Let us consider now the $1+2$ reaction in the laboratory frame, where the momentum of the target (the dimer) is 0 , and let us write the phase space $\Phi$ but using the momenta $\boldsymbol{p}_{i}$, $\boldsymbol{p}_{j}$, and $\boldsymbol{p}_{k}$ of the three particles after the breakup. Imposing energy and momentum conservation the phase space takes the form

$$
\begin{aligned}
\Phi= & \int d \boldsymbol{p}_{i} d \boldsymbol{p}_{j} d \boldsymbol{p}_{k} \delta\left(E_{\mathrm{lab}}-\frac{\hbar^{2} p_{i}^{2}}{2 m}-\frac{\hbar^{2} p_{j}^{2}}{2 m}-\frac{\hbar^{2} p_{k}^{2}}{2 m}\right) \\
& \times \delta^{3}\left(\boldsymbol{p}_{y}^{(\mathrm{lab})}-\boldsymbol{p}_{i}-\boldsymbol{p}_{j}-\boldsymbol{p}_{\boldsymbol{k}}\right),
\end{aligned}
$$

where $\boldsymbol{p}_{y}^{(\mathrm{lab})}$ is the momentum of the incoming particle in the laboratory frame, and $E_{\mathrm{lab}}$ is the total three-body energy in the laboratory frame,

$$
E_{\mathrm{lab}}=E_{\mathrm{in}}^{(\mathrm{lab})}+E_{d}=\frac{\hbar^{2} p_{y}^{(\mathrm{lab}) 2}}{2 m}+E_{d},
$$


where $E_{\text {in }}^{(\text {lab) }}$ is the energy of the projectile in the laboratory frame and $E_{d}$ is the binding energy of the dimer $\left(E_{d}<0\right)$. For the particular case of three particles with equal mass, the incident momentum in the laboratory frame is related to the one in the center-of-mass frame, $\boldsymbol{p}_{y}$, by the simple relation $\boldsymbol{p}_{y}^{(\mathrm{lab})}=3 \boldsymbol{p}_{y} / 2$.

Making use of the momentum $\delta$ function we integrate now over $\boldsymbol{p}_{k}$, which leads to

$$
\Phi=\int d \boldsymbol{p}_{i} d \boldsymbol{p}_{j} \delta\left(f\left(\boldsymbol{p}_{i}, \boldsymbol{p}_{j}\right)\right)
$$

where

$$
f\left(\boldsymbol{p}_{i}, \boldsymbol{p}_{j}\right)=E_{\mathrm{lab}}-\frac{\hbar^{2} p_{i}^{2}}{2 m}-\frac{\hbar^{2} p_{j}^{2}}{2 m}-\frac{\hbar^{2}\left(\boldsymbol{p}_{y}^{(\mathrm{lab})}-\boldsymbol{p}_{i}-\boldsymbol{p}_{j}\right)^{2}}{2 m} .
$$

Using Eq. (B7) the function above can be written as

$$
\begin{aligned}
f\left(\boldsymbol{p}_{i}, \boldsymbol{p}_{j}\right)= & E_{d}-\frac{\hbar^{2} p_{i}^{2}}{m}-\frac{\hbar^{2} p_{j}^{2}}{m} \\
& +\frac{\hbar^{2}}{m}\left(p_{y}^{(\mathrm{lab})} p_{i} \mu_{i}+p_{y}^{(\mathrm{lab})} p_{j} \mu_{j}-p_{i} p_{j} \mu\right),
\end{aligned}
$$

with

$$
\begin{aligned}
\mu_{i} & =\frac{\boldsymbol{p}_{y}^{(\mathrm{lab})} \cdot \boldsymbol{p}_{i}}{p_{y}^{(\mathrm{lab})} p_{i}}=\cos \theta_{p_{i}}, \\
\mu_{j} & =\frac{\boldsymbol{p}_{y}^{(\mathrm{lab})} \cdot \boldsymbol{p}_{j}}{p_{y}^{(\mathrm{lab})} p_{j}}=\cos \theta_{p_{j}}, \\
\mu & =\frac{\boldsymbol{p}_{i} \cdot \boldsymbol{p}_{j}}{p_{i} p_{j}}=\sin \theta_{p_{i}} \sin \theta_{p_{j}} \cos \Delta \varphi+\mu_{i} \mu_{j},
\end{aligned}
$$

where $\theta_{p_{i}}$ and $\theta_{p_{j}}$ are the polar angles associated with the direction of $\boldsymbol{p}_{i}$ and $\boldsymbol{p}_{j}$, respectively, and $\Delta \varphi$ is the difference between the corresponding azimuthal angles. The $z$ axis is chosen along the momentum $\boldsymbol{p}_{y}^{(\mathrm{lab})}$.

We now exploit the well-known property of the $\delta$ function,

$$
\delta(g(x))=\sum_{i} \frac{1}{\left|g^{\prime}\left(x_{i}\right)\right|} \delta\left(x-x_{i}\right)
$$

where the summation is over all the $x_{i}$ values satisfying $g\left(x_{i}\right)=$ 0 and where $g^{\prime}\left(x_{i}\right)$ is the value of the first derivative of $g(x)$ at point $x_{i}$. Thus getting the derivative of Eq. (B10) with respect to $p_{i}$ and using Eq. (B14), it is then easy to rewrite $\delta\left(f\left(\boldsymbol{p}_{i}, \boldsymbol{p}_{j}\right)\right)$ in a more convenient form and integrate Eq. (B8) over $p_{i}$, which leads to

$$
\Phi=\frac{m}{\hbar^{2}} \int p_{j}^{2} d p_{j} d \hat{\boldsymbol{p}}_{i} d \hat{\boldsymbol{p}}_{j} \frac{p_{i}^{2}}{\left|2 p_{i}-p_{y}^{(\mathrm{lab})} \mu_{i}+p_{j} \mu\right|},
$$

where, for each value of $p_{j}$ and the directions $\hat{\boldsymbol{p}}_{i}$ and $\hat{\boldsymbol{p}}_{j}, p_{i}$ is obtained as the solution of

$$
\begin{aligned}
& E_{d}-\frac{\hbar^{2} p_{i}^{2}}{m}-\frac{\hbar^{2} p_{j}^{2}}{m} \\
& +\frac{\hbar^{2}}{m}\left(p_{y}^{(\mathrm{lab})} p_{i} \mu_{i}+p_{y}^{(\mathrm{lab})} p_{j} \mu_{j}-p_{i} p_{j} \mu\right)=0 .
\end{aligned}
$$

In the same way it is possible to integrate Eq. (B8) over $p_{j}$, instead of $p_{i}$, and get

$$
\Phi=\frac{m}{\hbar^{2}} \int p_{i}^{2} d p_{i} d \hat{\boldsymbol{p}}_{i} d \hat{\boldsymbol{p}}_{j} \frac{p_{j}^{2}}{\left|2 p_{j}-p_{y}^{(\text {lab })} \mu_{j}+p_{i} \mu\right|} .
$$

From Eqs. (B15) and (B17) it is then clear that

$$
\frac{d p_{j}}{\left|2 p_{i}-p_{y}^{(\mathrm{lab})} \mu_{i}+p_{j} \mu\right|}=\frac{d p_{i}}{\left|2 p_{j}-p_{y}^{(\mathrm{lab})} \mu_{j}+p_{i} \mu\right|} .
$$

Since the energy $E_{i}$ of particle $i$ is given by $E_{i}=\hbar^{2} p_{i}^{2} / 2 m$ we have that $d E_{i}=\hbar^{2} p_{i} d p_{i} / m$ (and the same for particle $j$ ), and the expression above can be written as

$$
\frac{p_{i} d E_{j}}{\left|2 p_{i}-p_{y}^{(\mathrm{lab})} \mu_{i}+p_{j} \mu\right|}=\frac{p_{j} d E_{i}}{\left|2 p_{j}-p_{y}^{(\mathrm{lab})} \mu_{j}+p_{i} \mu\right|},
$$

or, in other words,

$$
d E_{j}=\frac{p_{j}\left|2 p_{i}-p_{y}^{(\mathrm{lab})} \mu_{i}+p_{j} \mu\right|}{p_{i}\left|2 p_{j}-p_{y}^{(\mathrm{lab})} \mu_{j}+p_{i} \mu\right|} d E_{i} .
$$

We now define the arclength $S$ such that

$$
d S=\sqrt{\left(d E_{i}\right)^{2}+\left(d E_{j}\right)^{2}},
$$

which, from Eq. (B20), leads to

$$
\begin{aligned}
& d E_{i}= \\
& \frac{p_{i}\left|2 p_{j}-p_{y}^{(\mathrm{lab})} \mu_{j}+p_{i} \mu\right| d S}{\left[p_{i}^{2}\left(2 p_{j}-p_{y}^{(\mathrm{lab})} \mu_{j}+p_{i} \mu\right)^{2}+p_{j}^{2}\left(2 p_{i}-p_{y}^{(\mathrm{lab})} \mu_{i}+p_{j} \mu\right)^{2}\right]^{1 / 2}} .
\end{aligned}
$$

Finally, keeping in mind that $E_{i}=\hbar^{2} p_{i}^{2} / 2 m$ and therefore $p_{i} d p_{i}=\left(m / \hbar^{2}\right) d E_{i}$, we can write the phase space, Eq. (B17), as

$$
\Phi=\left(\frac{m}{\hbar^{2}}\right)^{2} \int p_{i} d E_{i} d \hat{\boldsymbol{p}}_{i} d \hat{\boldsymbol{p}}_{j} \frac{p_{j}^{2}}{\left|2 p_{j}-p_{y}^{(\mathrm{lab})} \mu_{j}+p_{i} \mu\right|},
$$

which, by use of Eq. (B22), leads to the final expression for the phase space in the laboratory frame,

$$
d^{5} \Phi=\left(\frac{m}{\hbar^{2}}\right)^{2} K_{S} d S d \hat{\boldsymbol{p}}_{i} d \hat{\boldsymbol{p}}_{j}
$$

where

$$
\begin{aligned}
& K_{S}= \\
& \frac{p_{i}^{2} p_{j}^{2}}{\left[p_{i}^{2}\left(2 p_{j}-p_{y}^{(\mathrm{lab})} \mu_{j}+p_{i} \mu\right)^{2}+p_{j}^{2}\left(2 p_{i}-p_{y}^{(\mathrm{lab})} \mu_{i}+p_{j} \mu\right)^{2}\right]^{1 / 2}}
\end{aligned}
$$

\section{APPENDIX C: CALCULATION OF $p_{i}$ AND $p_{j}$ FOR A GIVEN INPUT}

In this work the $n-d$ breakup reaction is investigated taking as input the incident energy of the projectile in the laboratory frame $E_{\mathrm{in}}^{(\mathrm{lab})}$, the polar angles $\theta_{p_{i}}$ and $\theta_{p_{j}}$ of the 
two neutrons observed after the breakup, and the difference between the corresponding two azimuthal angles $\Delta \varphi$. From the energy $E_{\text {in }}^{(\mathrm{lab})}$ we can easily obtain the incident momentum of the projectile in the laboratory frame $\left[p_{y}^{(\mathrm{lab})}\right]$, the incident momentum in the center-of-mass frame $\left[p_{y}^{(\mathrm{in})}\right]$ and the threebody momentum $\kappa$ [see Eq. (16) and below]. The angles $\theta_{p_{i}}$, $\theta_{p_{j}}$, and $\Delta \varphi$ permit us to obtain $\mu_{i}, \mu_{j}$, and $\mu$, which are given by Eqs. (B11), (B12), and (B13), respectively.

The remaining point is to determine for each value of the arclength $S$ the values of $p_{i}$ and $p_{j}$, such that we can obtain $K_{S}$ according to Eq. (B25), and then obtain the cross section (15) as a function of $S$. The momenta $p_{i}$ and $p_{j}$ are not independent. They are related through expression (B16), which can be written as

$$
\alpha^{2}+p_{i}^{2}+p_{j}^{2}-p_{y}^{(\mathrm{lab})} p_{i} \mu_{i}-p_{y}^{(\mathrm{lab})} p_{j} \mu_{j}+p_{i} p_{j} \mu=0,
$$

where $\alpha^{2}=-m E_{d} / \hbar^{2} \quad\left(E_{d}<0\right)$. After some algebra the above equation can be seen to describe an ellipse, whose equation is given by

$$
\frac{\left(p_{i}^{\prime}-p_{i}^{C}\right)^{2}}{a_{i}^{2}}+\frac{\left(p_{j}^{\prime}-p_{j}^{C}\right)^{2}}{a_{j}^{2}}=1,
$$

where

$$
\begin{aligned}
& p_{i}^{C}=\frac{p_{y}^{(\mathrm{lab})}}{2 \sqrt{2}} \frac{\mu_{i}-\mu_{j}}{1-\mu / 2}, \\
& p_{j}^{C}=\frac{p_{y}^{(\mathrm{lab})}}{2 \sqrt{2}} \frac{\mu_{i}+\mu_{j}}{1+\mu / 2},
\end{aligned}
$$

and $a_{i}$ and $a_{j}$ are given as

$$
\begin{aligned}
& a_{i}=\frac{\beta}{\sqrt{1-\frac{\mu}{2}}}, \\
& a_{j}=\frac{\beta}{\sqrt{1+\frac{\mu}{2}}},
\end{aligned}
$$

with

$$
\beta^{2}=\frac{p_{y}^{(\mathrm{lab}) 2}}{8}\left[\frac{\left(\mu_{i}-\mu_{j}\right)^{2}}{1-\frac{\mu}{2}}+\frac{\left(\mu_{i}+\mu_{j}\right)^{2}}{1+\frac{\mu}{2}}\right]-\alpha^{2} .
$$

Note that, given the input, $p_{i}^{C}, p_{j}^{C}, a_{i}$, and $a_{j}$ are just numbers. Finally, $p_{i}^{\prime}$ and $p_{j}^{\prime}$ are such that

$$
\begin{aligned}
& p_{i}=\frac{p_{i}^{\prime}+p_{j}^{\prime}}{\sqrt{2}}, \\
& p_{j}=\frac{p_{j}^{\prime}-p_{i}^{\prime}}{\sqrt{2}} .
\end{aligned}
$$

The equation of the ellipse, (C2), can be written in parametric form as

$$
\begin{aligned}
& p_{i}^{\prime}-p_{i}^{C}=a_{i} \cos \tilde{\varphi}, \\
& p_{j}^{\prime}-p_{j}^{C}=a_{j} \sin \tilde{\varphi},
\end{aligned}
$$

which permits us to write Eqs. (C8) and (C9) as

$$
\begin{aligned}
& p_{i}=\frac{a_{i} \cos \tilde{\varphi}+p_{i}^{C}+a_{j} \sin \tilde{\varphi}+p_{j}^{C}}{\sqrt{2}}, \\
& p_{j}=\frac{a_{j} \sin \tilde{\varphi}+p_{j}^{C}-a_{i} \cos \tilde{\varphi}-p_{i}^{C}}{\sqrt{2}},
\end{aligned}
$$

from which we get

$$
\begin{aligned}
d p_{i} & =\frac{-a_{i} \sin \tilde{\varphi}+a_{j} \cos \tilde{\varphi}}{\sqrt{2}} d \tilde{\varphi}, \\
d p_{j} & =\frac{a_{j} \cos \tilde{\varphi}+a_{i} \sin \tilde{\varphi}}{\sqrt{2}} d \tilde{\varphi} .
\end{aligned}
$$

Remembering now that $d E_{i}=\hbar^{2} p_{i} d p_{i} / m$, and making use of the two equations above, we can write the arclength $S$ given in Eq. (B21) as

$$
d S=F(\tilde{\varphi}) d \tilde{\varphi}
$$

where

$$
\begin{aligned}
F(\tilde{\varphi})= & \frac{\hbar^{2}}{m} \frac{1}{\sqrt{2}}\left[p_{i}^{2}\left(a_{j} \cos \tilde{\varphi}-a_{i} \sin \tilde{\varphi}\right)^{2}\right. \\
& \left.+p_{j}^{2}\left(a_{j} \cos \tilde{\varphi}+a_{i} \sin \tilde{\varphi}\right)^{2}\right]^{1 / 2},
\end{aligned}
$$

in which $p_{i}$ and $p_{j}$ are given as a function of $\tilde{\varphi}$ by Eqs. (C12) and (C13), respectively. Therefore, the arclength $S$ can be obtained as a function of $\tilde{\varphi}$ from the expression

$$
S(\tilde{\varphi})=\int_{\tilde{\varphi}_{0}}^{\tilde{\varphi}} F\left(\tilde{\varphi}^{\prime}\right) d \tilde{\varphi}^{\prime},
$$

where, for all the cases except case $3, \tilde{\varphi}_{0}$ is defined such that for $\tilde{\varphi}=\tilde{\varphi}_{0}$ then $p_{j}=0$. For case 3 , since the ellipse in Eq. (C2) does not cross the $p_{j}=0$ axis, $\tilde{\varphi}_{0}$ is defined such that for $\tilde{\varphi}=\tilde{\varphi}_{0}$ then $p_{i}=0$.

In a practical case, given a value of $S$, one has to determine the angle $\tilde{\varphi}$ such that $S(\tilde{\varphi})=S$. With this value of $\tilde{\varphi}$ the values of $p_{i}$ and $p_{j}$ are immediately obtained from Eqs. (C12) and (C13).

\section{APPENDIX D: ADIABATIC EXPANSION OF THREE-BODY CONTINUUM WAVE FUNCTIONS}

In the Appendix of Ref. [22] the authors give the general solution of the coupled-channel problem for three-body continuum states in the presence of interaction potentials. Keeping the notation used in that reference, the continuum three-body wave function at a given three-body energy $E=\kappa^{2} \hbar^{2} / 2 m$ is written as

$$
\begin{aligned}
\Psi= & \sum_{J M} \frac{(2 \pi)^{3}}{(\kappa \rho)^{5 / 2}} \sum_{K \gamma, K^{\prime} \gamma^{\prime}} \psi_{K \gamma, K^{\prime} \gamma^{\prime}}^{J}(\kappa \rho) \Upsilon_{J M}^{K \gamma}\left(\Omega_{\rho}\right) \\
& \times \sum_{M_{L}^{\prime} M_{S}^{\prime}}\left\langle L^{\prime} M_{L}^{\prime} S^{\prime} M_{S}^{\prime} \mid J M\right\rangle \mathcal{Y}_{K^{\prime} L^{\prime} M_{L}^{\prime} \ell^{\prime}}\left(\Omega_{\kappa}\right)^{*},
\end{aligned}
$$

where the functions $\mathcal{Y}_{K^{\prime} L^{\prime} M_{L}^{\prime}}^{\ell_{x}^{\prime} \ell_{y}^{\prime}}$ are the usual HHs, the index $\gamma$ collects the quantum numbers $\left\{\ell_{x}, \ell_{y}, L, S\right\}$, and $\Upsilon_{J M}^{K \gamma}$ is defined 
as the coupling between the $\mathrm{HH} \mathcal{Y}_{K L M_{L}}^{\ell_{x} \ell_{y}}$ and the three-body spin function $\chi_{S M_{S}}$ :

$$
\Upsilon_{J M}^{K \gamma}\left(\Omega_{\rho}\right)=\sum_{M_{L} M_{S}}\left\langle L M_{L} S M_{S} \mid J M\right\rangle \mathcal{Y}_{K L M_{L}}^{\ell_{x} \ell_{y}}\left(\Omega_{\rho}\right) \chi_{S M_{S}} .
$$

It is convenient to write the total three-body wave function as $\Psi=\sum_{S^{\prime} M_{S}^{\prime}} \Psi_{S^{\prime} M_{S}^{\prime}}$, where

$$
\begin{aligned}
\Psi_{S^{\prime} M_{S}^{\prime}}= & \sum_{J M} \frac{(2 \pi)^{3}}{(\kappa \rho)^{5 / 2}} \sum_{K \gamma} \sum_{K^{\prime} \ell_{x}^{\prime} \ell_{y}^{\prime} L^{\prime}} \psi_{K \gamma, K^{\prime} \gamma^{\prime}}^{J}(\kappa \rho) \Upsilon_{J M}^{K \gamma}\left(\Omega_{\rho}\right) \\
& \times \sum_{M_{L}^{\prime}}\left\langle L^{\prime} M_{L}^{\prime} S^{\prime} M_{S}^{\prime} \mid J M\right\rangle \mathcal{Y}_{K^{\prime} L^{\prime} M_{L}^{\prime}}^{\ell_{x}^{\prime} \ell_{y}^{\prime}}\left(\Omega_{\kappa}\right)^{*},
\end{aligned}
$$

which represents the continuum three-body wave function where the spin function of the incoming channel is described by the quantum numbers $S^{\prime}$ and $M_{S}^{\prime}$.

This can be easily seen because, in the case of no interaction between the particles, the radial wave functions $\psi_{K \gamma, K^{\prime} \gamma^{\prime}}^{J}(\kappa \rho)$ reduce to $i^{K} \sqrt{\kappa \rho} J_{K+2}(\kappa \rho) \delta_{K K^{\prime}} \delta_{\gamma, \gamma^{\prime}}$, and the three-body wave function becomes

$$
\Psi_{S^{\prime} M_{S}^{\prime}} \stackrel{\text { no interaction }}{\longrightarrow} e^{i\left(\boldsymbol{k}_{x} \cdot \boldsymbol{x}+\boldsymbol{k}_{y} \cdot \boldsymbol{y}\right)} \chi_{S^{\prime} M_{S}^{\prime}},
$$

which is a three-body plane wave multiplied by the threebody spin function in the incoming channel. Now using the expression Eq. (D2) the wave function in Eq. (D3) can be written in a more compact way as

$$
\begin{aligned}
\Psi_{S^{\prime} M_{S}^{\prime}}= & \sum_{J M} \frac{(2 \pi)^{3}}{(\kappa \rho)^{5 / 2}} \sum_{K \gamma} \sum_{K^{\prime} \ell_{x}^{\prime} \ell_{y}^{\prime} L^{\prime}} \psi_{K \gamma, K^{\prime} \gamma^{\prime}}^{J}(\kappa \rho) \Upsilon_{J M}^{K \gamma}\left(\Omega_{\rho}\right) \\
& \times\left\langle\chi_{S^{\prime} M_{S}^{\prime}} \mid \Upsilon_{J M}^{K^{\prime} \gamma^{\prime}}\left(\Omega_{\kappa}\right)\right\rangle^{*} .
\end{aligned}
$$

The transformation of the wave function $\Psi_{S^{\prime}} M_{S}^{\prime}$ from the $\mathrm{HH}$ basis to the basis formed by the hyperangular functions $\Phi_{n}^{J M}$ defined in Eq. (19) can be easily made through the relation

$$
\Upsilon_{J M}^{K \gamma}=\sum_{n}\left\langle\Phi_{n}^{J M} \mid \Upsilon_{J M}^{K \gamma}\right\rangle \Phi_{n}^{J M},
$$

which leads to

$$
\begin{aligned}
\Psi_{S^{\prime} M_{S}^{\prime}}= & \sum_{J M} \frac{(2 \pi)^{3}}{(\kappa \rho)^{5 / 2}} \\
& \times \sum_{n n^{\prime}} f_{n n^{\prime}}^{S^{\prime} J}(\kappa \rho) \Phi_{n}^{J M}\left(\rho, \Omega_{\rho}\right)\left\langle\chi_{S^{\prime} M_{S}^{\prime}}\left|\Phi_{n^{\prime}}^{J M}\left(\kappa, \Omega_{\kappa}\right)\right|^{*},\right.
\end{aligned}
$$

and where we have defined

$$
\begin{aligned}
f_{n n^{\prime}}^{S^{\prime} J(\kappa \rho)}= & \left.\sum_{K \gamma} \sum_{K^{\prime} \ell_{x}^{\prime} \ell_{y}^{\prime} L^{\prime}} \psi_{K \gamma, K^{\prime} \gamma^{\prime}}^{J}(\kappa \rho)\left|\Phi_{n}^{J M}\left(\rho, \Omega_{\rho}\right)\right| \Upsilon_{J M}^{K \gamma}\left(\Omega_{\rho}\right)\right\rangle \\
& \times\left\langle\Upsilon_{J M}^{K^{\prime} \gamma^{\prime}}\left(\Omega_{\kappa}\right) \mid \Phi_{n^{\prime}}^{J M}\left(\kappa, \Omega_{\kappa}\right)\right\rangle .
\end{aligned}
$$

As shown in Eq. (D4), the expansion in Eq. (D1) has been written in such a way that, in the case of no interaction between the particles, the wave function, Eq. (D3), reduces basically to a three-body plane. In fact, the way they are written, Eq. (D1) or (D3) is appropriate to describe $3 \rightarrow 3$ reactions and would permit us to extract the corresponding $3 \rightarrow 3$ transition amplitude.

However, when dealing with $1+2$ reactions, in the case of no interaction between the projectile and the dimer, the three-body continuum wave function must reduce to

$$
\Psi_{\sigma_{d} \sigma_{p}} \stackrel{\text { no interaction }}{\longrightarrow} e^{i \boldsymbol{k}_{y} \cdot \boldsymbol{y}} \chi_{s_{d} \sigma_{d}} \chi_{s_{p} \sigma_{p}},
$$

where $\chi_{s_{d} \sigma_{d}}$ is the dimer wave function (with spin $s_{d}$ and projection $\sigma_{d}$ ), and $\chi_{s_{p} \sigma_{p}}$ is the spin function of the projectile. As we can see, the three-body plane wave is now an ordinary two-body plane wave (describing the relative free motion between the projectile and the dimer center of mass). In order to obtain such a two-body plane wave with the correct normalization the factor $(2 \pi)^{3}$ in the expansion in Eq. (D1), and therefore also in the following expressions of the continuum wave function, has to be replaced with $(2 \pi)^{3 / 2}$.

Also, as seen from Eq. (D9), if we want to describe the incoming spin state by $\chi_{s_{d} \sigma_{d}} \chi_{s_{p} \sigma_{p}}$ (instead of the total threebody spin and projection $S^{\prime}$ and $M_{S}^{\prime}$ ), the corresponding wave function $\Psi_{\sigma_{d} \sigma_{p}}$ can be obtained from $\Psi_{S^{\prime} M_{S}^{\prime}}$ through the simple relation

$$
\Psi_{\sigma_{d} \sigma_{p}}=\sum_{S^{\prime} M_{S}^{\prime}}\left\langle\chi_{S^{\prime} M_{S}^{\prime}} \mid \sigma_{p} \sigma_{d}\right\rangle \Psi_{S^{\prime} M_{S}^{\prime}}
$$

where $\left|\sigma_{p} \sigma_{d}\right\rangle$ represents the spin state $\left|\chi_{s_{d} \sigma_{d}} \chi_{s_{p} \sigma_{p}}\right\rangle$, and from which we can write

$$
\begin{aligned}
\Psi_{\sigma_{d} \sigma_{p}}= & \sum_{J M} \frac{(2 \pi)^{3 / 2}}{(\kappa \rho)^{5 / 2}} \sum_{n n^{\prime}} f_{n n^{\prime}}^{J}(\kappa \rho) \Phi_{n}^{J M}\left(\rho, \Omega_{\rho}\right) \\
& \times\left\langle\sigma_{d} \sigma_{p}\left|\Phi_{n^{\prime}}^{J M}\left(\kappa, \Omega_{\kappa}\right)\right|^{*}\right.
\end{aligned}
$$

where

$$
f_{n n^{\prime}}^{J}(\kappa \rho)=\sum_{S^{\prime} M_{S}^{\prime}}\left\langle\chi_{S^{\prime} M_{S}^{\prime}} \mid \sigma_{d} \sigma_{p}\right\rangle f_{n n^{\prime}}^{S^{\prime} J}(\kappa \rho)\left\langle\sigma_{d} \sigma_{p} \mid \chi_{S^{\prime} M_{S}^{\prime}}\right\rangle
$$

and where we have assumed that the final radial wave functions do not depend on the spin projections and that the interaction does not mix different spin states $\left|\sigma_{d} \sigma_{p}\right\rangle$.

As usual, in Eq. (D11) the indices $n$ and $n^{\prime}$ refer to the outgoing and incoming channels, respectively, and Eq. (D11) gives the full three-body wave function, where all possible incoming and outgoing channels are contained. Only the spin projections in the incoming channels are specified. If we now consider a specific incoming channel, say channel 1 , the summation over $n^{\prime}$ disappears, and $n^{\prime}=1$. Also, if we want to restrict ourselves to outgoing breakup channels, the summation over $n$ should run only over the adiabatic terms associated with breakup of the dimer. In particular, for the case in which only one $1+2$ channel exists (incoming channel 1), we have that the summation over $n$ runs over all $n>1$.

Finally, if we project over a specific spin state $\left|\sigma_{i} \sigma_{j} \sigma_{k}\right\rangle$ for the three particles after the breakup, we then get the final expression for the adiabatic expansion of the three-body wave function describing the breakup of the dimer, and it is given by

$$
\begin{aligned}
\Psi_{\sigma_{d} \sigma_{p}}^{\sigma_{i} \sigma_{j} \sigma_{k}}= & \sum_{J M} \frac{(2 \pi)^{3 / 2}}{(\kappa \rho)^{5 / 2}} \sum_{n>1} f_{n 1}^{J}(\kappa \rho) \\
& \times\left\langle\sigma_{i} \sigma_{j} \sigma_{k} \mid \Phi_{n}^{J M}\left(\rho, \Omega_{\rho}\right)\right\rangle\left\langle\sigma_{d} \sigma_{p}\left|\Phi_{n^{\prime}}^{J M}\left(\kappa, \Omega_{\kappa}\right)\right|^{*} .\right.
\end{aligned}
$$




\section{APPENDIX E: ASYMPTOTIC INCOMING ANGULAR EIGENFUNCTION IN MOMENTUM SPACE}

In coordinate space, the angular eigenfunction $\Phi_{1}\left(\rho, \Omega_{\rho}\right)$ associated with a $1+2$ channel behaves, for large values of $\rho$, as [1]

$$
\begin{aligned}
& \Phi_{1}^{J M}\left(\rho, \Omega_{\rho}\right) \\
& \stackrel{\rho \rightarrow \infty}{\rightarrow}\left(\frac{m}{\mu_{x}}\right)^{3 / 4} \rho^{3 / 2}\left[\psi_{d}^{j_{x}}\left(\boldsymbol{r}_{x}\right) \otimes\left[Y_{\ell_{y}}\left(\Omega_{y}\right) \otimes \chi_{s_{y}}\right]^{j_{y}}\right]^{J M},
\end{aligned}
$$

where $\psi_{d}^{j_{x}}\left(\boldsymbol{r}_{x}\right)$ is the dimer wave function, which is normalized to 1 in the relative coordinate $\boldsymbol{r}_{x}$ between the two particles in the dimer and whose angular momentum is denoted by $j_{x}$. The projectile-dimer relative orbital angular momentum $\ell_{y}$ and the spin of the projectile $s_{y}$ couple to the total angular momentum $j_{y}$, which in turn couples to $j_{x}$ to provide the total angular momentum $J$ of the three-body system, whose projection is given by $M$. Finally, $\mu_{x}$ is the reduced mass of the two particles in the dimer.

After a Fourier transformation the dimer wave function becomes $\psi_{d}^{j_{x}}\left(\boldsymbol{p}_{x}\right)$, also normalized to 1 in $\boldsymbol{p}_{x}$, where $\boldsymbol{p}_{x}$ is the relative momentum between the two particles in the dimer [Eq. (5)]. Also, the polar and azimuthal angles $\Omega_{y}$ describing the direction of the Jacobi coordinate $\boldsymbol{y}$ become $\Omega_{k_{y}}$, which describe the direction of the incident Jacobi momentum $\boldsymbol{k}_{y}^{(\mathrm{in})}$ defined as in Eq. (6). Therefore, the Fourier transform of Eq. (E1) must have the form

$$
\Phi_{1}^{J M}\left(\kappa, \Omega_{\kappa}\right) \rightarrow C\left[\psi_{d}^{j_{x}}\left(\boldsymbol{p}_{x}\right) \otimes\left[Y_{\ell_{y}}\left(\Omega_{k_{y}}\right) \otimes \chi_{s_{y}}\right]^{j_{y}}\right]^{J M},
$$

where $C$ is a normalization constant.

The value of $C$ can be obtained by imposing that

$$
\int d \Omega_{\kappa}\left|\Phi_{1}\left(\kappa, \Omega_{\kappa}\right)\right|^{2}=1
$$

which, after using the expansion

$$
\Psi_{d}^{j_{x} m_{x}}\left(\boldsymbol{p}_{x}\right)=\sum_{\ell_{x} s_{x}} \Psi_{\ell_{x} s_{x}}^{j_{x}}\left(p_{x}\right)\left[Y_{\ell_{x}}\left(\Omega_{k_{x}}\right) \otimes \chi_{s_{x}}\right]^{j_{x} m_{x}},
$$

leads to

$$
|C|^{2} \int d \alpha_{\kappa} \sin ^{2} \alpha_{\kappa} \cos ^{2} \alpha_{\kappa} \sum_{\ell_{x} s_{x}}\left|\Psi_{\ell_{x} s_{x}}^{j_{x}}\left(p_{x}\right)\right|^{2}=1
$$

Using now that $k_{x}=\kappa \sin \alpha_{\kappa}$ (and therefore $d k_{x}=$ $\kappa \cos \alpha_{\kappa} d \alpha_{\kappa}$ ) and $k_{y}^{(\text {in) }}=\kappa \cos \alpha_{\kappa}$, the expression above can be rewritten as

$$
|C|^{2} \frac{k_{y}^{(\mathrm{in})}}{\kappa^{4}} \int d k_{x} k_{x}^{2} \sum_{\ell_{x} s_{x}}\left|\Psi_{\ell_{x} s_{x}}^{j_{x}}\left(p_{x}\right)\right|^{2}=1
$$

which, after taking into account that $k_{x}^{2} d k_{x}=$ $\left(m / \mu_{x}\right)^{3 / 2} p_{x}^{2} d p_{x}$, and keeping in mind that $\psi_{d}^{j_{x}}\left(\boldsymbol{p}_{x}\right)$ is normalized to 1 in $\boldsymbol{p}_{x}$, leads to

$$
|C|^{2}=\frac{\kappa^{4}}{k_{y}^{(\mathrm{in})}}\left(\frac{\mu_{x}}{m}\right)^{3 / 2},
$$

and therefore,

$$
\begin{aligned}
\Phi_{1}^{J M}\left(\kappa, \Omega_{\kappa}\right) \rightarrow & \frac{\kappa^{2}}{\sqrt{k_{y}^{(\mathrm{in})}}}\left(\frac{\mu_{x}}{m}\right)^{3 / 4} \\
& \times\left[\psi_{d}^{j_{x}}\left(\boldsymbol{p}_{x}\right) \otimes\left[Y_{\ell_{y}}\left(\Omega_{k_{y}}\right) \otimes \chi_{s_{y}}\right]^{j_{y}}\right]^{J M} .
\end{aligned}
$$

In the particular case of only relative $s$ waves between the particles, the expression above reduces to

$$
\begin{aligned}
\Phi_{1}^{J M}\left(\kappa, \Omega_{\kappa}\right) \rightarrow & \frac{1}{\sqrt{4 \pi}}\left(\frac{\mu_{x}}{m}\right)^{3 / 4} \frac{\kappa^{2}}{\sqrt{k_{y}^{(\mathrm{in})}}} \\
& \times\left[\psi_{d}^{s_{d}}\left(\boldsymbol{p}_{x}\right) \otimes \chi_{s_{p}}\right]^{J M},
\end{aligned}
$$

where we have recovered the notation in Sec. IIC, where $s_{d}$ represents the spin of the dimer and $s_{p}$ is the spin of the projectile. From Eq. (E9) it is now simple to see that the projection over the intrinsic states of projectile and target, the asymptotic incoming angular wave function in momentum space, is given by

$$
\left\langle\sigma_{d} \sigma_{p} \mid \Phi_{1}^{*}\left(\kappa, \Omega_{\kappa}\right)\right\rangle=\frac{1}{\sqrt{4 \pi}}\left(\frac{\mu_{x}}{m}\right)^{3 / 4} \frac{\kappa^{2}}{\sqrt{k_{y}^{(\mathrm{in})}}}\left\langle s_{d} \sigma_{d} s_{p} \sigma_{p} \mid J M\right\rangle
$$

which is the expression given in Eq. (25).

\section{APPENDIX F：FRESNEL INTEGRALS}

The aim of this section is to compute analytically the integral,

$$
\int_{y_{\max }}^{\infty} d y\left(\frac{C_{m} \sin (\kappa y) \sin \left(k_{y} y\right)}{y^{m+3 / 2}}+\frac{D_{m} \cos (\kappa y) \sin \left(k_{y} y\right)}{y^{m+3 / 2}}\right),
$$

which can also be written as

$$
\begin{aligned}
& \frac{C_{m}}{2} \int_{y_{\max }}^{\infty} d y \frac{\cos \left(\left(\kappa-k_{y}\right) y\right)-\cos \left(\left(\kappa+k_{y}\right) y\right)}{y^{m+3 / 2}} \\
& -\frac{D_{m}}{2} \int_{y_{\max }}^{\infty} d y \frac{\sin \left(\left(\kappa-k_{y}\right) y\right)-\sin \left(\left(\kappa+k_{y}\right) y\right)}{y^{m+3 / 2}} .
\end{aligned}
$$

Therefore, the calculation of the integrals above requires just the calculation of the integrals of the type

$$
\int_{y_{\max }}^{\infty} d y \frac{\cos (a y)}{y^{m+3 / 2}} \text { and } \int_{y_{\max }}^{\infty} d y \frac{\sin (a y)}{y^{m+3 / 2}},
$$

where $a$ can be either $\left(\kappa-k_{y}\right)$ or $\left(\kappa+k_{y}\right)$. Now taking $t=a y$ the two integrals above take the form

$$
a^{m+1 / 2} \int_{t_{\max }}^{\infty} d t \frac{\cos t}{t^{m+3 / 2}} \text { and } a^{m+1 / 2} \int_{t_{\max }}^{\infty} d t \frac{\sin t}{t^{m+3 / 2}},
$$

where $t_{\max }=a y_{\max }$.

Let us now define

$$
\begin{aligned}
& \tilde{S}_{v}(z)=\int_{z}^{\infty} d x \frac{\sin x}{x^{v}}, \\
& \tilde{C}_{v}(z)=\int_{z}^{\infty} d x \frac{\cos x}{x^{v}},
\end{aligned}
$$


such that the two integrals in Eq. (F4) are $\tilde{C}_{3 / 2}\left(t_{\max }\right)$ and $\tilde{S}_{3 / 2}\left(t_{\max }\right)$, respectively. If we now take into account that

$$
\begin{aligned}
& \frac{\sin x}{x^{\nu}} d x=\frac{1}{v-1} \frac{\cos x}{x^{\nu-1}} d x-\frac{1}{v-1} d\left(\frac{\sin x}{x^{\nu-1}}\right), \\
& \frac{\cos x}{x^{\nu}} d x=-\frac{1}{v-1} \frac{\sin x}{x^{\nu-1}} d x-\frac{1}{v-1} d\left(\frac{\cos x}{x^{\nu-1}}\right),
\end{aligned}
$$

we can immediately get the recurrence relations:

$$
\begin{aligned}
& \tilde{S}_{v}(z)=\frac{1}{v-1} \frac{\sin z}{z^{v-1}}+\frac{1}{v-1} \tilde{C}_{v-1}(z), \\
& \tilde{C}_{v}(z)=\frac{1}{v-1} \frac{\cos z}{z^{v-1}}-\frac{1}{v-1} \tilde{S}_{v-1}(z) .
\end{aligned}
$$

Therefore, knowledge of $\tilde{S}_{1 / 2}(z)$ and $\tilde{C}_{1 / 2}(z)$ would permit us, through the recurrence relations in Eqs. (F9) and (F10), to obtain the integrals in Eq. (F3) and, therefore, the integral in Eq. (F2) [or (F1)].

The integrals $\tilde{S}_{1 / 2}(z)$ and $\tilde{C}_{1 / 2}(z)$ are known analytically, and they are given by

$$
\begin{aligned}
& \tilde{S}_{1 / 2}(z)=\sqrt{\frac{\pi}{2}}-\frac{\sqrt{\pi}}{2}(\sqrt{i} \operatorname{erf}(\sqrt{i z})+\sqrt{-i} \operatorname{erf}(\sqrt{-i z})), \\
& \tilde{C}_{1 / 2}(z)=\sqrt{\frac{\pi}{2}}-\frac{\sqrt{\pi}}{2}(\sqrt{-i} \operatorname{erf}(\sqrt{-i z})+\sqrt{i} \operatorname{erf}(\sqrt{i z})),
\end{aligned}
$$

where "erf" stands for the usual error function.
[1] E. Nielsen, D. V. Fedorov, A. S. Jensen, and E. Garrido, Phys. Rep. 347, 373 (2001).

[2] P. Barletta and A. Kievsky, Few-Body Syst. 45, 25 (2009).

[3] P. Barletta, C. Romero-Redondo, A. Kievsky, M. Viviani, and E. Garrido, Phys. Rev. Lett. 103, 090402 (2009).

[4] C. Romero-Redondo, E. Garrido, P. Barletta, A. Kievsky, and M. Viviani, Phys. Rev. A 83, 022705 (2011).

[5] A. Kievsky, M. Viviani, P. Barletta, C. Romero-Redondo, and E. Garrido, Phys. Rev. C 81, 034002 (2010).

[6] E. Garrido, C. Romero-Redondo, A. Kievsky, and M. Viviani, Phys. Rev. A 86, 052709 (2012).

[7] E. Garrido, M. Gattobigio, and A. Kievsky, Phys. Rev. A 88, 032701 (2013).

[8] G. L. Payne, W. Glöckle, and J. L. Friar, Phys. Rev. C 61, 024005 (2000).

[9] W. Glöckle, H. Witała, D. Hüber, H. Kamada, and J. Golak, Phys. Rep. 274, 107 (1996).

[10] J. Carlson and R. Schiavilla, Rev. Mod. Phys. 70, 743 (1998).

[11] J. Carbonell, A. Deltuva, A. C. Fonseca, and R. Lazauskas, Prog. Part. Nucl. Phys. 74, 55 (2014).
[12] A. Kievsky, M. Viviani, and S. Rosati, Phys. Rev. C 64, 024002 (2001).

[13] A. Deltuva, A. C. Fonseca, and P. U. Sauer, Phys. Rev. C 71, 054005 (2005)

[14] A. Deltuva, A. C. Fonseca, A. Kievsky, S. Rosati, P. U. Sauer, and M. Viviani, Phys. Rev. C 71, 064003 (2005).

[15] A. Kievsky, M. Viviani, and S. Rosati, Phys. Rev. C 56, 2987 (1997).

[16] A. Kievsky, S. Rosati, and M. Viviani, Phys. Rev. Lett. 82, 3759 (1999).

[17] M. Viviani, A. Kievsky, and S. Rosati, Few-Body Syst. 30, 39 (2001).

[18] J. L. Friar, G. L. Payne, W. Glöckle, D. Hüber, and H. Witala, Phys. Rev. C 51, 2356 (1995).

[19] A. Cobis, D. V. Fedorov, and A. S. Jensen, Phys. Rev. C 58, 1403 (1998).

[20] J. L. Friar et al., Phys. Rev. C 42, 1838 (1990).

[21] A. Deltuva, A. C. Fonseca, and P. U. Sauer, Phys. Rev. C 72, 054004 (2005).

[22] B. V. Danilin, T. Rogde, J. S. Vaagen, I. J. Thompson, and M. V. Zhukov, Phys. Rev. C 69, 024609 (2004). 